\title{
A Fuzzy Asymmetric TOPSIS model for Optimizing Investment in Online Advertising Campaigns
}

\author{
Francisco-Javier Arroyo-Cañada* (Department of Business, University of Barcelona ) \\ Jaime Gil-Lafuente (Department of Business, University of Barcelona ) \\ * Address of correspondence: Av. Diagonal, 690 Office 2324; e-mail: fjarroyo@ub.edu
}

\begin{abstract}
The high penetration of the Internet and e-commerce in Spain during recent years has increased companies' interest in this medium for advertising planning. In this context Google offers a great advertising inventory and perfectly segmented content pages.

This work is concerned with the optimization of online advertising investments based on payper-click campaigns. Our main goal is to rank and select different alternative keyword sets aimed at maximizing the awareness of and traffic to a company's website. The keyword selection problem with online advertising purposes is clearly a multiple-criteria decisionmaking problem additionally characterized by the imprecise, ambiguous and uncertain nature of the available data. To address this problem, we propose a TOPSIS-based approach, which allows us to rank the alternative keyword sets, taking into account the fuzzy nature of the available data. The Technique for Order of Preference by Similarity to Ideal Solution (TOPSIS) is based on the concept that the chosen alternative should have the shortest distance from the positive ideal solution (PIS) and the longest distance from the negative ideal solution (NIS). In this work, due to the characteristics of the studied problem, we propose the use of an asymmetric distance, allowing us to work with ideal solutions that differ from the maximum or the minimum. The suitability of the proposed model is illustrated with an empirical case of a stock exchange broker's advertising investment problem aimed at generating awareness about the brand and increasing the traffic to the corporative website.
\end{abstract}

Keywords: fuzzy, multiple-criteria decision making, online advertising, stock exchange 


\section{Introduction}

The Internet is changing the way in which companies try to connect with their target audience. The penetration rate of the Internet in Spain was 78.69\% in 2015 (ITU 2016). On the other hand, e-commerce has increased, with year-on-year growth of $20.3 \%$ during the second quarter of 2016, according to the Spanish National Authority for Markets and Competition (CNMC 2017).

The possibility of accessing millions of consumers has made the Internet the fastest-growing advertising medium in Spain according to Infoadex (2017). Consequently, many companies allocate part of their promotional investments to this medium. It is therefore not surprising that stock exchange brokers use this channel to attract potential customers looking for information on this type of service.

Google offers access to a great advertising (henceforth ad) inventory in many perfectly segmented content pages. The Display Network inventory is composed of different ad formats (texts, images, animation or video) and different locations within the set of web pages that Google controls. This inventory can be accessed using a pay-per-click system for a set of keywords through the Google AdWords tool. Given the millions of potential sites for advertising insertions, advertisers face a complex multiple-criteria decision-making problem to select the best alternatives to connect with their target groups effectively and efficiently.

We focus on the case of the advertising investment of a stock exchange broker with two main goals: generating awareness about the brand and increasing the traffic to the corporative website. The decision maker faces the maximization of clicks, impressions per week, cookies per week, opportunity to see (OTS), click-through rate (CTR) and relevance, at the same time as minimizing the average cost per click (CPC) of several alternative sets of keywords for the advertising campaign. The awareness is related to impressions per week, cookies per week, OTS and relevance, while the traffic is most linked with clicks, CTR and CPC. At the same time, there may be criteria for which a maximum or minimum point is not the positive or negative ideal, for example the case of the OTS, in which the experts fix an intermediate point as the positive ideal. Then it is convenient to resolve a common problem with the distance measures to the ideal related to the penalization of excesses and lacks.

To solve the problem of the optimization of the investment in ads, an adaptation of the Technique for Order of Preference by Similarity to Ideal Solution (TOPSIS) is proposed. The model allows us to rank the different alternatives of keyword sets to optimize the investment 
in online advertising of a stock exchange brokerage service taking into account two different goals, several decision-making criteria and the uncertainty and imprecision of the available data. In addition, one of the novelties of our approach is the consideration of an asymmetric distance function, which will allow us to consider ideal solutions that are different from the maximum or the minimum values.

The remainder of this paper is structured as follows. Section 2 provides a brief literature review about advertising planning, highlighting the multiple-criteria nature of this kind of problem. The proposed new method based on TOPSIS is presented in Section 3. Section 4 presents and discusses a real case study to show the suitability of the proposed approach. The paper ends with some conclusions in Section 5.

\section{The multiple criteria nature of the online advertising planning problem}

The great penetration of the Internet has turned a website into a useful tool to access information in customers' purchase decision-making process. An increasing number of researchers acknowledge the important role of the Internet from different viewpoints. Some authors focus on the determinants of customer interactions with e-banking (Liao and Wong 2008), others concentrate on the influence of the online reputation on the purchase process (Kim et al. 2008) and several authors study the role of the Internet in advertising planning for bank services (Fruchter and Dou 2005).

The growth of e-commerce has made the Internet a lucrative place for advertising (Kumar and Sethi 2009). Search-based advertising has become very popular, since it provides advertisers with the ability to attract potential customers with measurable returns (Selçuk and Özlük 2013).

In the Google search engine, two types of results are shown: organic and sponsored. The organic search results are based on the relevance of the websites computed by the ranking algorithm of the search engine. In this sense companies must develop a search engine optimization strategy (SEO) to gain a good position in Google's search results. On the other side, a search engine marketing strategy (SEM) allows companies to select related keywords to show their advertising in the result pages of user searches. In this way the advertising becomes relevant to users. Analogously, the Google Display Network enhances the advertising inventory with other web pages, such as blogs, forums, virtual communities or content websites. 
Regarding online advertising planning, Anderson (2006) and Grappone and Couzin (2008) propose the use of keywords that are specialized in market niches instead of expending advertising efforts on a few very popular markets. Brooks (2004), Feng et al. (2007) and Regelson and Fain (2006) study the relationship between ad position and clicks.

Several authors also address the keyword-bidding problem. Feldman et al. (2007) introduce the notion of a click price curve, in which the clicks increase as the cost per click rises. Zhou et al. (2008) consider bidding optimization under budget restrictions. In the same way, Özlük and Cholette (2007) optimize the keywords considering the click-through rate (CTR) of the ad placement and a cost-profit analysis.

Zhao and Nagurney (2008) examine advertising bidding, in which multiple firms compete for advertising spaces on multiple websites. Selçuk and Özlük (2013) propose two different models to minimize the advertising cost while guaranteeing a desirable level of exposure. In the first case, the exposure is measured by the average CTR weighted by the number of impressions of all the keywords used in the campaign, and, in the second case, the exposure is measured by the total number of impressions weighted by the relative ad positions of all the keywords.

From the above brief literature survey, we can conclude that online advertising is clearly a complex multiple-criteria decision-making problem. Within the MCDM approaches, TOPSIS is shown to be a practical and useful technique for ranking and selecting a number of determined alternatives through distance measures (Shih et al. 2007). As mentioned above, TOPSIS is a simple ranking method, which identifies the alternatives that simultaneously have the shortest distance from the positive ideal solution and the farthest distance from the negative ideal solution.

This method is shown to work satisfactorily in a large number of application areas (see Behzadian et al. (2012) for a review). Its success is based on several features that make it very attractive for real problems: TOPSIS's logic is rational and understandable; the computation processes are straightforward; the concept permits the pursuit of the best alternatives for each criterion depicted in a simple mathematical form; and the importance weights are incorporated into the comparison procedures (see García-Cascales and Lamata 2012).

However, the method has some drawbacks. One of them is the reverse-order phenomenon (see for example Saaty and Vargas 1984; Saaty 1987; Saaty and Vargas 1993; Saaty and Sagir 2009). In this phenomenon the order of the alternatives changes when an alternative is added 
to or eliminated from the decision problem. The use of the absolute PIS and NIS can contribute to solving the above-mentioned limitation (Ceballos et al. 2013). Another weakness is the consistency checking for judgements. However, in this research the decision matrix is completed with statistical information provided by Google instead of being based on the judgements of experts or decision makers.

Another characteristic of the online advertising decision-making problem is the uncertain, ambiguous and imprecise nature of the available data. Fuzzy set theory (Zadeh 1965) is a suitable tool to handle the fuzziness characterizing real-world decision-making problems, and it has been applied successfully in a large number of areas, such as production planning and scheduling, location, transportation, finance and engineering design. TOPSIS has also benefited from fuzzy set theory to incorporate ambiguity, uncertainty and imprecision (see Nadaban et al. (2016) for a recent review of the development of fuzzy TOPSIS methods and applications).

\section{Methodology: Fuzzy asymmetric TOPSIS approach}

Let us consider a stock exchange brokerage service that plans to invest in online advertising with two main goals: generating brand awareness and increasing the traffic to its website. In this context one of the key questions is the selection of keyword sets in a search engine marketing campaign (SEM) in Google AdWords. In the selection of the best keyword sets, the following decision-making criteria are taken into account for both goals:

Advertising inventory is the total emplacements into which the advertising can be inserted in the Google Display Network (e.g. blogs, forums, content pages and other websites that use Google AdSense to gain extra income). In this case the inventory is related to the cookies per week and determines how many people are interested in the proposed keywords. The advertising is only shown to interested people who visit the inventory of the Google Display Network.

Impressions per week is the estimated number of visualizations that the advertising receives in a week (i.e. the number of downloads from the website into which the advertising is inserted).

Clicks per week is the number of times that users click on the advertising (this involves major premeditation of the user).

Opportunity to see (OTS) is the average number of impressions that users receive. In this sense the cookies identify different users. 
Click-through rate (CTR) is an effectiveness measure of advertising. A higher CTR is evidence of the attractiveness of the message.

Cost per click measures the average price of the advertising for the keyword.

Relevance of the advertising place is related to the affinity of the message to the context. For example, the advertising of a stock exchange brokerage service within a blog related to stock investment strategies is more relevant.

In the following we will describe the different steps of the proposed algorithm adapted to the problem addressed in this paper:

Step 1. Complete the decision matrix from data provided by the advertising planner tool of Google.

\begin{tabular}{|c|c|c|c|c|}
\hline & $\boldsymbol{C}_{\boldsymbol{1}}$ & $\boldsymbol{C}_{\mathbf{2}}$ & $\ldots$ & $\boldsymbol{C}_{\boldsymbol{n}}$ \\
\hline $\boldsymbol{A}_{\boldsymbol{1}}$ & $x_{11}$ & $x_{12}$ & $\ldots$ & $x_{1 n}$ \\
\hline $\boldsymbol{A}_{\boldsymbol{2}}$ & $x_{21}$ & $x_{22}$ & $\ldots$ & $x_{2 n}$ \\
\hline$\ldots$ & $\ldots$ & $\ldots$ & $\ldots$ & $\ldots$ \\
\hline $\boldsymbol{A}_{\boldsymbol{m}}$ & $x_{m 1}$ & $x_{m 2}$ & $\ldots$ & $x_{m n}$ \\
\hline
\end{tabular}

where $A_{i}$ denotes alternative $i, i=1, \ldots, m ; x_{j}$ represents the attribute or criterion $j, j=1, \ldots$, $n$; and $x_{i j}$ indicates the performance rating of alternative $A_{i}$ with respect to attribute $x_{j}$. The values of $x_{i j}$ can be replaced with fuzzy numbers or linguistic values to introduce fuzzy indicators.

Step 2. Normalize the decision matrix.

To work with the same scales for all the attributes, it is convenient to normalize the decision matrix. Several normalization procedures are possible, for example vector normalization, linear normalization or non-monotonic normalization. In this work we have chosen vector normalization, because it is simple and is the most used in the literature (see García-Cascales and Lamata 2012).

$$
n_{i j}=\frac{x_{i j}}{\sqrt{\sum_{j=1}^{m}\left(x_{i j}\right)^{2}}} \quad i=1, \ldots, m ; j=1, \ldots, n
$$

Step 3. Weight the normalized decision matrix.

It is possible that attributes do not have the same importance in the aggregation process. Then it is necessary to multiply the normalized matrix by the weighting coefficients: 


$$
v_{i j}=w_{j} \times n_{i j} \quad i=1, \ldots, m ; j=1, \ldots, n
$$

Step 4. Determine the PIS $\left(A^{+}\right)$and NIS $\left(A^{-}\right)$.

The positive and negative ideal values are determined in the following way (see GarcíaCascales and Lamata 2012):

$$
\begin{aligned}
& A^{+}=\left\{v_{1}^{+}, \ldots, v_{1}^{+}\right\}=\left\{\left(\max v_{i j}, j \in J\right)\left(\min v_{i j}, j \in J^{\prime}\right)\right\} \\
& A^{-}=\left\{v_{1}^{-}, \ldots, v_{1}^{-}\right\}=\left\{\left(\min v_{i j}, j \in J\right)\left(\max v_{i j}, j \in J^{\prime}\right)\right\}
\end{aligned}
$$

where $J$ is associated with benefit criteria (the more the better) and $J$ ' is associated with cost criteria (the fewer the better).

Step 5. Calculate distances to PIS $\left(A^{+}\right)$and NIS $\left(A^{-}\right)$.

TOPSIS usually uses measures based on Minkowski's distance (Hamming $p=1$, Euclidean $p$ $=2$ and Tchebycheff $p=\alpha$ ) to measure the proximity of each alternative to the PIS and NIS.

$$
\begin{aligned}
& d_{i}^{+}=\left\{\sum_{j=1}^{n}\left|v_{i j}-v_{j}^{+}\right|^{p}\right\}^{\frac{1}{p}} ; i=1, \ldots, m \\
& d_{i}^{-}=\left\{\sum_{j=1}^{n}\left|v_{i j}-v_{j}^{-}\right|^{p}\right\}^{\frac{1}{p}} ; i=1, \ldots, m
\end{aligned}
$$

In particular, Hamming's distance can be considered as a particular case of Minkowski's distance (Merigó and Gil-Lafuente 2012). This is expressed as:

$$
\begin{aligned}
& d_{i}^{+}=\sum_{j=1}^{n}\left|v_{i j}-v_{j}^{+}\right| ; i=1, \ldots, m \\
& d_{i}^{-}=\sum_{j=1}^{n}\left|v_{i j}-v_{j}^{-}\right| ; i=1, \ldots, m
\end{aligned}
$$

However, one can wonder what happens when the ideal is not a maximum or minimum value. A common problem with the distance measures (Euclidean, Hamming and Tchebycheff) is the question related to the penalization of excesses and lacks. To resolve this problem, we propose an asymmetric TOPSIS, with a distance measure that is not linear and different penalties for excesses and lacks, considering the weighted Hamming's distance as an asymmetric distance (Dong et al. 2008). Let us introduce a characteristic membership function $\mu_{A_{+}}\left(x_{j}\right)$ for the distance measure to penalize the distance to the ideal for the attribute (j). This function involves an asymmetric weighting system that penalizes the excesses of frequency more than the lacks. When the keyword set's expectation is below the ideal, this is considered to be a worse situation than excess. However, we need to distinguish between a light excess and a hard excess. 
It is possible to generalize the distance expression as:

$$
\begin{aligned}
& d_{i}\left(A, A^{+}\right)=\sum_{j=1}^{n}\left|v_{i j}-v_{j}^{+}\right|^{\mu_{A_{+}}\left(x_{j}\right)}, \text { for alternative } i, i=1, \ldots, m . \\
& d_{i}\left(A, A^{-}\right)=\sum_{j=1}^{n}\left|v_{i j}-v_{j}^{-}\right|^{\mu_{A_{-}}\left(x_{j}\right)}, \text { for alternative } i, i=1, \ldots, m .
\end{aligned}
$$

This is an original contribution of the authors to introduce an asymmetric weighting system to penalize the distance to the ideal.

Step 6. Calculate the relative proximity to the ideal solution.

$$
R_{i}=\frac{d_{i}^{-}}{d_{i}^{+}+d_{i}^{-}} ; i=1, \ldots, m
$$

Step 7. Rank the alternatives in descending order by the proximity to the ideal solution.

A set of alternatives can now be preference ranked according to the descending order of the value of $R_{i}$.

\section{Empirical example}

We will now illustrate the proposed approach using a real example. Let us consider a stock exchange brokerage service that intends to optimize its investment in online advertisement. The decision maker has two different goals: maximizing the awareness and maximizing the traffic to the company's website. Twenty-four different alternative keyword sets have been selected with the aim of satisfying the two considered goals (see Table 1).

For this example all the criteria have a maximum or minimum as the positive or negative ideal with the exception of the opportunity to see (OTS). In this case the positive ideal is fixed by the decision maker in the range $x_{4}=[5,10]$. Values lower than 5 are penalized more than values higher than 10. In the same way, values higher than 20 are penalized more than values between 10 and 20. In the case of the advertising decision maker, the characteristic membership function $\mu_{A_{+}}\left(x_{j}\right)$ takes the following values:

$$
\begin{aligned}
& \mu_{A_{+}}\left(x_{4}\right)=1 ; \mu_{A_{-}}\left(x_{4}\right)=0.2 \forall x_{4} \in[5,10] \\
& \mu_{A_{+}}\left(x_{4}\right)=0.2 ; \mu_{A_{-}}\left(x_{4}\right)=1 \quad \forall x_{4}<5 \\
& \mu_{A_{+}}\left(x_{4}\right)=0.7 ; \mu_{A_{-}}\left(x_{4}\right)=0.5 \forall x_{4} \in[10,20] \\
& \mu_{A_{+}}\left(x_{4}\right)=0.5 ; \mu_{A_{-}}\left(x_{4}\right)=0.7 \forall x_{4}>20
\end{aligned}
$$


For other attributes it is not necessary to penalize the distance to the positive or negative ideal; then $\mu_{A_{+}}\left(x_{j}\right)=1 ; \mu_{A_{-}}\left(x_{j}\right)=1 \forall j \neq 4$ (OTS criterion).

Table 1. Alternative keyword sets

\begin{tabular}{|c|c|}
\hline Alternatives & Keywords set \\
\hline $\mathrm{A}_{1}$ & financial services, investment services, finance, investment \\
\hline $\mathrm{A}_{2}$ & $\begin{array}{l}\text { broker for investment in stock exchange, platform for investment in stock exchange, stock } \\
\text { market values, companies for investment }\end{array}$ \\
\hline $\mathrm{A}_{3}$ & $\begin{array}{l}\text { investment in the stock exchange, how invest in stock exchange, stock exchange shares, how is } \\
\text { it the stock exchange, the stock exchange }\end{array}$ \\
\hline $\mathrm{A}_{4}$ & $\begin{array}{l}\text { stock exchange broker, investment stock exchange, stock exchange market, stock exchange } \\
\text { quotes, stock exchange forex, options, stock exchange operations, foreign exchange, trading, } \\
\text { stock exchange and trading, CFD, stock exchange platform, stock exchange for beginners }\end{array}$ \\
\hline $\mathrm{A}_{5}$ & $\begin{array}{l}\text { investment in the stock exchange, investment stock exchange, stock exchange, Spain stock } \\
\text { exchange, stock exchange market }\end{array}$ \\
\hline $\mathrm{A}_{6}$ & investment in stock exchange, how investment in stock exchange \\
\hline $\mathrm{A}_{7}$ & stock exchange broker, futures, stock exchange agent, \\
\hline$A_{8}$ & $\begin{array}{l}\text { stocks in the stock exchange, how to trade, win money in the stock exchange, win in the stock } \\
\text { exchange, how win in the stock exchange }\end{array}$ \\
\hline $\mathrm{A}_{9}$ & $\begin{array}{l}\text { learn to invest in stock market, invest in stock market, invest in stock market beginners, values } \\
\text { in stock market investing, investing in stock market online, In which to invest in stock market }\end{array}$ \\
\hline $\mathrm{A}_{10}$ & $\begin{array}{l}\text { how to invest money in stock exchange, invest in the stock exchange, stock exchange ibex } \\
\text { spanish }\end{array}$ \\
\hline$A_{11}$ & values of the stock exchange, companies of the stock exchange \\
\hline $\mathrm{A}_{12}$ & stock exchange \\
\hline $\mathrm{A}_{13}$ & buy in stock exchange \\
\hline $\mathrm{A}_{14}$ & stock exchange beginners, stock exchange rookie \\
\hline $\mathrm{A}_{15}$ & invest money in the stock exchange, in stock exchange \\
\hline $\mathrm{A}_{16}$ & how to invest in stock exchange for beginners, how to invest in the stock market \\
\hline $\mathrm{A}_{17}$ & broker forex, broker forex trading, forex broker forex trading \\
\hline $\mathrm{A}_{18}$ & broker of forex \\
\hline $\mathrm{A}_{19}$ & $\begin{array}{l}\text { cfd broker, trading broker, brokers cfds, trading online broker, best broker cfd, forex broker, } \\
\text { broker options, broker foreign exchange, cfd online broker }\end{array}$ \\
\hline $\mathrm{A}_{20}$ & $\begin{array}{l}\text { stock exchange money, stock exchange broker, gold stock exchange, online stock exchange, the } \\
\text { stock exchanges, stock exchange profitability }\end{array}$ \\
\hline $\mathrm{A}_{21}$ & stock exchange \\
\hline $\mathrm{A}_{22}$ & cfd trader \\
\hline $\mathrm{A}_{23}$ & real time online stock exchange, real time stock exchange \\
\hline $\mathrm{A}_{24}$ & foreign exchange, stock exchange of \\
\hline
\end{tabular}

The key planner tool of Google AdWords allows the completion of the information about the attributes of each alternative. Table 2 displays the resulting decision matrix and Table 3 the normalized decision matrix.

Table 2. Decision matrix

\begin{tabular}{cccccccc}
\hline $\begin{array}{c}\text { Keyword } \\
\text { sets }\end{array}$ & Clicks & $\begin{array}{c}\text { Impressions } \\
\text { week }\end{array}$ & $\begin{array}{c}\text { Cookies } \\
\text { week }\end{array}$ & OTS & CTR & $\begin{array}{c}\text { Average } \\
\text { CPC }\end{array}$ & Relevance \\
\hline $\mathrm{A}_{1}$ & 2.920 & 1.123 .077 & {$[100.000,500.000]$} & {$\left[\begin{array}{llll}2.25, & 11.23\end{array}\right]$} & 0.0026 & 0.61 & 10 \\
$\mathrm{~A}_{2}$ & 91 & 65.000 & {$[35.000,40.000]$} & {$\left[\begin{array}{llll}1.63, & 1.86\end{array}\right]$} & 0.0014 & 0.50 & 10
\end{tabular}




\begin{tabular}{|c|c|c|c|c|c|c|c|c|}
\hline $\mathrm{A}_{3}$ & 46.000 & 9.787 .234 & {$[1.500 .000$} & $2.000 .000]$ & {$[4.89,6.52]$} & 0.0047 & 0.10 & 9 \\
\hline $\mathrm{A}_{4}$ & 43.500 & 6.041 .667 & {$[2.000 .000$,} & $2.500 .000]$ & {$[2.42,3.02]$} & 0.0072 & 0.10 & 8 \\
\hline $\mathrm{A}_{5}$ & 36.300 & 4.321 .429 & {$[1.500 .000$,} & $2.000 .000]$ & {$[2.16,2.88]$} & 0.0084 & 0.07 & 8 \\
\hline $\mathrm{A}_{6}$ & 63.500 & 13.510 .638 & {$[2.500 .000$,} & $3.000 .000]$ & {$[4.50,5.40]$} & 0.0047 & 0.11 & 7 \\
\hline $\mathrm{A}_{7}$ & 39.700 & 5.925 .373 & {$[1.500 .000$,} & $2.000 .000]$ & {$[2.96,3.95]$} & 0.0067 & 0.06 & 7 \\
\hline $\mathrm{A}_{8}$ & 40.100 & 7.160 .714 & {$[2.000 .000$,} & $2.500 .000]$ & {$[2.86,3.58]$} & 0.0056 & 0.09 & 6 \\
\hline $\mathrm{A}_{9}$ & 65.800 & 13.428 .571 & {$[2.000 .000$, } & $2.500 .000]$ & {$[5.37,6.71]$} & 0.0049 & 0.10 & 6 \\
\hline $\mathrm{A}_{10}$ & 19 & 1.776 & {$[1.000$} & $1.500]$ & {$[1.18,1.78]$} & 0.0107 & 0.84 & 5 \\
\hline $\mathrm{A}_{11}$ & 42.600 & 4.580 .645 & {$[1.500 .000$,} & $2.000 .000]$ & {$[2.29,3.05]$} & 0.0093 & 0.06 & 4 \\
\hline $\mathrm{A}_{12}$ & 361 & 361.000 & {$[200.000$} & $250.000]$ & {$[1.44,1.81]$} & 0.0010 & 0.33 & 4 \\
\hline$A_{13}$ & 64.100 & 12.094 .340 & {$[250.000$} & $300.000]$ & {$[40.31,48.38]$} & 0.0053 & 0.09 & 4 \\
\hline $\mathrm{A}_{14}$ & 45 & 16.667 & {$[5.000$} & $10.000]$ & {$[1.67,3.33]$} & 0.0027 & 0.50 & 3 \\
\hline $\mathrm{A}_{15}$ & 170 & 106.250 & {$[50.000$,} & $100.000]$ & {$[1.06,2.13]$} & 0.0016 & 0.19 & 3 \\
\hline $\mathrm{A}_{16}$ & 9 & 4.737 & {$[2.500$} & $3.000]$ & {$[1.58,1.89]$} & 0.0019 & 0.52 & 2 \\
\hline$A_{17}$ & 28.100 & 1.243 .363 & {$[250.000$} & $300.000]$ & {$[4.14,4.97]$} & 0.0226 & 0.04 & 1 \\
\hline $\mathrm{A}_{18}$ & 27.300 & 1.087 .649 & {$[200.000$} & $250.000]$ & {$[4.35,5.44]$} & 0.0251 & 0.04 & 1 \\
\hline $\mathrm{A}_{19}$ & 33.200 & 2.862 .069 & {$[500.000$} & $1.000 .000]$ & {$[2.86,5.72]$} & 0.0116 & 0.06 & 1 \\
\hline $\mathrm{A}_{20}$ & 18.000 & 4.090 .909 & {$[1.000 .000$,} & $1.500 .000]$ & {$[2.73,4.09]$} & 0.0044 & 0.12 & 1 \\
\hline $\mathrm{A}_{21}$ & 36.900 & 3.324 .324 & [1.000.000, & $1.500 .000]$ & {$[2.22,3.32]$} & 0.0111 & 0.07 & 1 \\
\hline $\mathrm{A}_{22}$ & 438 & 336.923 & {$[150.000$} & 200.000] & {$[1.68,2.25]$} & 0.0013 & 0.52 & 1 \\
\hline $\mathrm{A}_{23}$ & 122 & 33.889 & {$[20.000$} & 25.000] & {$[1.36,1.69]$} & 0.0036 & 0.41 & 1 \\
\hline $\mathrm{A}_{24}$ & 42 & 35.000 & {$[15.000$} & 20.000] & {$[1.75,2.33]$} & 0.0012 & 0.24 & 1 \\
\hline PIS A+ & 65.800 & 13.510 .638 & {$[2.500 .000$,} & $3.000 .000]$ & {$[5,10]$} & 0.025 & 0.04 & 10 \\
\hline NIS A- & 9 & 1776 & {$[1000$} & 1500] & {$[0,0]$} & 0.001 & 0.84 & 1 \\
\hline
\end{tabular}

Table 3. Normalized decision matrix

\begin{tabular}{|c|c|c|c|c|c|c|c|c|c|}
\hline \multirow{2}{*}{$\begin{array}{c}\begin{array}{c}\text { Keyword } \\
\text { sets }\end{array} \\
\mathrm{A}_{1}\end{array}$} & \multirow{2}{*}{$\begin{array}{c}\text { Clicks } \\
0.018\end{array}$} & \multirow{2}{*}{$\begin{array}{c}\begin{array}{c}\text { Impressions } \\
\text { week }\end{array} \\
0.040\end{array}$} & \multicolumn{2}{|c|}{$\begin{array}{c}\text { Cookies } \\
\text { week }\end{array}$} & \multicolumn{2}{|c|}{ OTS } & \multirow{2}{*}{$\begin{array}{l}\text { CTR } \\
0.059\end{array}$} & \multirow{2}{*}{$\begin{array}{c}\begin{array}{c}\text { Average } \\
\text { CPC }\end{array} \\
0.379\end{array}$} & \multirow{2}{*}{$\begin{array}{c}\text { Relevance } \\
0.383\end{array}$} \\
\hline & & & {$[0.018$} & $0.071]$ & {$[0.053$,} & $0.212]$ & & & \\
\hline $\mathrm{A}_{2}$ & 0.001 & 0.002 & {$[0.006$} & $0.006]$ & {$[0.035$,} & $0.038]$ & 0.032 & 0.311 & 0.383 \\
\hline $\mathrm{A}_{3}$ & 0.279 & 0.344 & {$[0.275$,} & $0.283]$ & {$[0.115$,} & $0.123]$ & 0.106 & 0.062 & 0.345 \\
\hline $\mathrm{A}_{4}$ & 0.264 & 0.213 & {$[0.354$,} & $0.367]$ & {$[0.057$,} & $0.057]$ & 0.163 & 0.062 & 0.306 \\
\hline $\mathrm{A}_{5}$ & 0.220 & 0.152 & {$[0.275$} & $0.283]$ & {$[0.051$,} & $0.054]$ & 0.190 & 0.044 & 0.306 \\
\hline $\mathrm{A}_{6}$ & 0.386 & 0.475 & {$[0.425$} & $0.458]$ & {$[0.102$,} & $0.106]$ & 0.106 & 0.068 & 0.268 \\
\hline $\mathrm{A}_{7}$ & 0.241 & 0.208 & {$[0.275$} & $0.283]$ & {$[0.070$} & $0.075]$ & 0.152 & 0.037 & 0.268 \\
\hline $\mathrm{A}_{8}$ & 0.243 & 0.252 & {$[0.354$} & $0.367]$ & {$[0.067$,} & $0.068]$ & 0.127 & 0.056 & 0.230 \\
\hline $\mathrm{A}_{9}$ & 0.400 & 0.473 & {$[0.354$} & $0.367]$ & {$[0.126$} & $0.127]$ & 0.111 & 0.062 & 0.230 \\
\hline $\mathrm{A}_{10}$ & 0.000 & 0.000 & {$[0.000$} & $0.000]$ & {$[0.028$} & $0.034]$ & 0.242 & 0.522 & 0.191 \\
\hline $\mathrm{A}_{11}$ & 0.259 & 0.161 & {$[0.275$} & $0.283]$ & {$[0.054$} & $0.058]$ & 0.210 & 0.037 & 0.153 \\
\hline $\mathrm{A}_{12}$ & 0.002 & 0.013 & {$[0.035$} & $0.037]$ & {$[0.034$} & $0.034]$ & 0.023 & 0.205 & 0.153 \\
\hline $\mathrm{A}_{13}$ & 0.389 & 0.426 & {$[0.042$} & $0.046]$ & {$[0.915$} & $0.947]$ & 0.120 & 0.056 & 0.153 \\
\hline $\mathrm{A}_{14}$ & 0.000 & 0.001 & {$[0.001$} & $0.001]$ & {$[0.039$} & $0.063]$ & 0.061 & 0.311 & 0.115 \\
\hline $\mathrm{A}_{15}$ & 0.001 & 0.004 & {$[0.009$} & $0.014]$ & {$[0.025$,} & $0.040]$ & 0.036 & 0.118 & 0.115 \\
\hline $\mathrm{A}_{16}$ & 0.000 & 0.000 & {$[0.000$} & $0.000]$ & {$[0.036$} & $0.037]$ & 0.043 & 0.323 & 0.077 \\
\hline $\mathrm{A}_{17}$ & 0.171 & 0.044 & {$[0.042$,} & $0.046]$ & {$[0.094$,} & $0.097]$ & 0.511 & 0.025 & 0.038 \\
\hline $\mathrm{A}_{18}$ & 0.166 & 0.038 & {$[0.035$} & $0.037]$ & {$[0.102$,} & $0.103]$ & 0.568 & 0.025 & 0.038 \\
\hline $\mathrm{A}_{19}$ & 0.202 & 0.101 & {$[0.092$} & $0.142]$ & {$[0.067$,} & $0.108]$ & 0.262 & 0.037 & 0.038 \\
\hline $\mathrm{A}_{20}$ & 0.109 & 0.144 & {$[0.183$} & $0.212]$ & {$[0.064$,} & $0.077]$ & 0.100 & 0.075 & 0.038 \\
\hline $\mathrm{A}_{21}$ & 0.224 & 0.117 & {$[0.183$} & $0.212]$ & {$[0.052$} & $0.063]$ & 0.251 & 0.044 & 0.038 \\
\hline $\mathrm{A}_{22}$ & 0.003 & 0.012 & {$[0.028$} & $0.028]$ & {$[0.040$} & $0.042]$ & 0.029 & 0.323 & 0.038 \\
\hline $\mathrm{A}_{23}$ & 0.001 & 0.001 & {$[0.004$} & $0.004]$ & {$[0.032$} & $0.032]$ & 0.081 & 0.255 & 0.038 \\
\hline $\mathrm{A}_{24}$ & 0.000 & 0.001 & {$[0.003$} & $0.003]$ & {$[0.041$,} & $0.044]$ & 0.027 & 0.149 & 0.038 \\
\hline PIS A+ & 0.400 & 0.475 & {$[0.425$} & $0.458]$ & {$[0.117$,} & $0.189]$ & 0.566 & 0.025 & 0.383 \\
\hline
\end{tabular}


Weights reflecting the different degrees of importance of the different criteria for each goal are obtained directly from the decision maker (see Table 4).

Table 4. Criteria weighting coefficients for each of the goals

\begin{tabular}{cccccccc}
\hline Goal & Clicks & Impressions & Cookies & OTS & CTR & CPC & Relevance \\
\hline Traffic & 0.185 & 0.130 & 0.130 & 0.148 & 0.185 & 0.093 & 0.130 \\
Awareness & 0.123 & 0.175 & 0.175 & 0.140 & 0.123 & 0.123 & 0.140 \\
\hline
\end{tabular}

The next step consists of calculating the distance to the PIS and NIS. As previously mentioned, the decision maker has two different goals. In this work we use a linear combination of the distance functions to the PIS and NIS:

$$
\begin{aligned}
& d_{i}\left(A, A^{+}\right)=\propto_{j} \sum_{j=1}^{n}\left|v_{i j}-v_{j}^{+}\right|^{\mu_{A_{+}}\left(x_{j}\right)}+\left(1-\propto_{j}\right) \sum_{j=1}^{n}\left|v_{i j}-v_{j}^{+}\right|^{\mu_{A_{+}}\left(x_{j}\right)} \\
& d_{i}\left(A, A^{-}\right)=\propto_{k} \sum_{j=1}^{n}\left|v_{i j}-v_{j}^{-}\right|^{\mu_{A_{-}}\left(x_{j}\right)}+\left(1-\propto_{k}\right) \sum_{j=1}^{n}\left|v_{i j}-v_{j}^{-}\right|^{\mu_{A_{-}}\left(x_{j}\right)}
\end{aligned}
$$

Table 5 displays the distance results for equal weights $\left(\propto_{j}=\propto_{k}=0.5\right)$. The first part of the table displays the results for the fuzzy TOPSIS case (using the Euclidean distance and not penalizing slacks from the ideal solution). The second part of the table displays the results obtained with the fuzzy asymmetric approach proposed in this paper, which penalizes slacks from the ideal solution.

\begin{tabular}{|c|c|c|c|c|c|c|}
\hline \multirow{2}{*}{$\begin{array}{c}\text { Keyword } \\
\text { sets }\end{array}$} & \multicolumn{3}{|c|}{ Fuzzy TOPSIS } & \multicolumn{3}{|c|}{ Fuzzy and asymmetric TOPSIS } \\
\hline & $d_{i}\left(A, A^{-}\right)$ & $d_{i}\left(A, A^{+}\right)$ & $R_{i}$ & $d_{i}\left(A, A^{-}\right)$ & $d_{i}\left(A, A^{+}\right)$ & $R_{i}$ \\
\hline $\mathrm{A}_{1}$ & {$[0.003,0.003]$} & {$[0.029,0.037]$} & {$[0.079,0.081]$} & {$[0.411,0.569]$} & {$[0.397,0.438]$} & {$[0.509,0.565]$} \\
\hline $\mathrm{A}_{2}$ & {$[0.003,0.003]$} & {$[0.038,0.039]$} & {$[0.064,0.067]$} & {$[0.072,0.074]$} & {$[0.972,0.982]$} & {$[0.069,0.070]$} \\
\hline $\mathrm{A}_{3}$ & {$[0.011,0.011]$} & {$[0.020,0.021]$} & {$[0.346,0.362]$} & {$[0.661,0.663]$} & {$[0.254,0.266]$} & {$[0.713,0.722]$} \\
\hline $\mathrm{A}_{4}$ & {$[0.010,0.010]$} & {$[0.022,0.023]$} & {$[0.310,0.318]$} & {$[0.238,0.241]$} & {$[0.806,0.815]$} & {$[0.228,0.228]$} \\
\hline $\mathrm{A}_{5}$ & {$[0.008,0.008]$} & {$[0.023,0.024]$} & {$[0.256,0.267]$} & {$[0.216,0.217]$} & {$[0.827,0.839]$} & {$[0.206,0.207]$} \\
\hline $\mathrm{A}_{6}$ & {$[0.017,0.018]$} & {$[0.019,0.020]$} & {$[0.468,0.469]$} & {$[0.688,0.705]$} & {$[0.210,0.214]$} & {$[0.766,0.767]$} \\
\hline $\mathrm{A}_{7}$ & {$[0.008,0.009]$} & {$[0.022,0.024]$} & {$[0.266,0.278]$} & {$[0.220,0.221]$} & {$[0.823,0.835]$} & {$[0.210,0.211]$} \\
\hline $\mathrm{A}_{8}$ & {$[0.009,0.010]$} & {$[0.022,0.023]$} & {$[0.301,0.294]$} & {$[0.227,0.230]$} & {$[0.817,0.825]$} & {$[0.217,0.218]$} \\
\hline $\mathrm{A}_{9}$ & {$[0.016,0.016]$} & {$[0.019,0.020]$} & {$[0.448,0.459]$} & {$[0.700,0.708]$} & {$[0.220,0.228]$} & {$[0.757,0.761]$} \\
\hline $\mathrm{A}_{10}$ & {$[0.002,0.002]$} & {$[0.036,0.038]$} & {$[0.041,0.043]$} & {$[0.055,0.055]$} & {$[0.990,1001]$} & {$[0.052,0.052]$} \\
\hline $\mathrm{A}_{11}$ & {$[0.008,0.008]$} & {$[0.023,0.024]$} & {$[0.249,0.260]$} & {$[0.207,0.208]$} & {$[0.837,0.848]$} & {$[0.197,0.198]$} \\
\hline $\mathrm{A}_{12}$ & {$[0.001,0.001]$} & {$[0.037,0.039]$} & {$[0.036,0.038]$} & {$[0.058,0.059]$} & {$[0.987,0.997]$} & {$[0.055,0.056]$} \\
\hline $\mathrm{A}_{13}$ & {$[0.027,0.029]$} & {$[0.009,0.010]$} & {$[0.743,0.744]$} & {$[0.448,0.456]$} & {$[0.171,0.175]$} & {$[0.723,0.724]$} \\
\hline
\end{tabular}

Table 5. Distances to the PIS and NIS and proximities to the ideal solution 


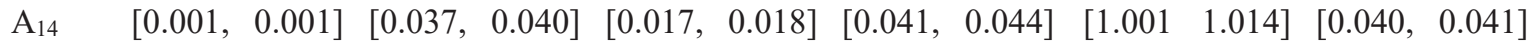

$\mathrm{A}_{15} \quad[0.002,0.002][0.037,0.040][0.049,0.052][0.058,0.060][0.985,0.998][0.056,0.057]$

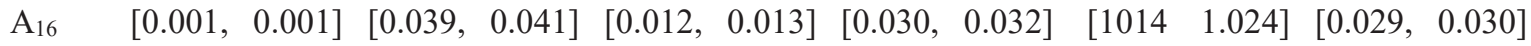

$\mathrm{A}_{17}[0.010,0.010][0.025,0.027][0.265,0.276][0.177,0.179][0.867,0.876][0.170,0.170]$

$\mathrm{A}_{18} \quad[0.011,0.011][0.025,0.027][0.291,0.304][0.574,0.581][0.326,0.336][0.634,0.638]$

$\mathrm{A}_{19}[0.006,0.006][0.024,0.028][0.177,0.190][0.510,0.563][0.342,0.365][0.599,0.607]$

$\mathrm{A}_{20}[0.004,0.004][0.028,0.030][0.127,0.129][0.132,0.138][0.907,0.923][0.127,0.130]$

$\mathrm{A}_{21} \quad[0.006,0.007][0.025,0.027][0.198,0.206][0.171,0.176][0.869,0.885][0.164,0.166]$

$\mathrm{A}_{22}[0.000,0.000][0.039,0.041][0.012,0.013][0.030,0.031][1.014,1.025][0.029,0.029]$

$\mathrm{A}_{23}[0.001,0.001][0.038,0.040][0.023,0.024][0.039,0.040][1.006,1.016][0.037,0.038]$ $\mathrm{A}_{24}[0.002,0.002][0.039,0.041][0.039,0.041][0.043,0.044][1.001,1.012][0.041,0.042]$

Finally, we order the alternatives by their proximity to the ideal solution, taking into account the range average. In this case alternative $\mathrm{A}_{6}$ occupies the best option for the advertising campaign. Concretely, the keyword set composed of "investment in stock exchange and how to investment in stock exchange" is at the top of the ranking.

Table 6. Rankings of proximities to the ideal solution

\begin{tabular}{|c|c|c|c|}
\hline Keywords set & $\begin{array}{c}\text { Proximity } R_{i} \\
\text { (Fuzzy TOPSIS) }\end{array}$ & Keywords set & $\begin{array}{c}\text { Proximity } R_{i} \\
\text { (Fuzzy and asymmetric } \\
\text { TOPSIS) }\end{array}$ \\
\hline $\mathrm{A}_{13}$ & {$[0.743,0.744]$} & $\mathrm{A}_{6}$ & {$\left[\begin{array}{ll}0.766, & 0.767\end{array}\right]$} \\
\hline $\mathrm{A}_{6}$ & {$[0.468,0.469]$} & $\mathrm{A}_{9}$ & {$[0.757,0.761]$} \\
\hline $\mathrm{A}_{9}$ & {$[0.448,0.459]$} & $\mathrm{A}_{13}$ & {$[0.723,0.724]$} \\
\hline $\mathrm{A}_{3}$ & {$[0.346,0.362]$} & $\mathrm{A}_{3}$ & {$[0.713,0.722]$} \\
\hline $\mathrm{A}_{4}$ & {$[0.310,0.318]$} & $\mathrm{A}_{18}$ & {$[0.634,0.638]$} \\
\hline $\mathrm{A}_{8}$ & {$[0.294,0.301]$} & $\mathrm{A}_{19}$ & {$[0.599,0.607]$} \\
\hline $\mathrm{A}_{18}$ & {$[0.291,0.304]$} & $\mathrm{A}_{1}$ & {$[0.509,0.565]$} \\
\hline $\mathrm{A}_{7}$ & {$[0.266,0.278]$} & $\mathrm{A}_{4}$ & {$[0.228,0.228]$} \\
\hline $\mathrm{A}_{17}$ & {$[0.265,0.276]$} & $\mathrm{A}_{8}$ & {$[0.217,0.218]$} \\
\hline $\mathrm{A}_{5}$ & {$[0.256,0.267]$} & $\mathrm{A}_{7}$ & {$[0.210,0.211]$} \\
\hline $\mathrm{A}_{11}$ & {$[0.249,0.260]$} & $\mathrm{A}_{5}$ & {$[0.206,0.207]$} \\
\hline $\mathrm{A}_{21}$ & {$[0.198,0.206]$} & $\mathrm{A}_{11}$ & {$[0.197,0.198]$} \\
\hline $\mathrm{A}_{19}$ & {$[0.177,0.190]$} & $\mathrm{A}_{17}$ & {$[0.170,0.170]$} \\
\hline $\mathrm{A}_{20}$ & {$[0.127,0.129]$} & $\mathrm{A}_{21}$ & {$[0.164,0.166]$} \\
\hline $\mathrm{A}_{1}$ & {$[0.079,0.081]$} & $\mathrm{A}_{20}$ & {$[0.127,0.130]$} \\
\hline $\mathrm{A}_{2}$ & {$[0.064,0.067]$} & $\mathrm{A}_{2}$ & {$[0.069,0.070]$} \\
\hline $\mathrm{A}_{15}$ & {$[0.049,0.052]$} & $\mathrm{A}_{15}$ & {$[0.056,0.057]$} \\
\hline $\mathrm{A}_{10}$ & {$[0.041,0.043]$} & $\mathrm{A}_{12}$ & {$[0.055,0.056]$} \\
\hline $\mathrm{A}_{24}$ & {$[0.039,0.041]$} & $\mathrm{A}_{10}$ & {$[0.052,0.052]$} \\
\hline $\mathrm{A}_{12}$ & {$[0.036,0.038]$} & $\mathrm{A}_{24}$ & {$[0.041,0.042]$} \\
\hline $\mathrm{A}_{23}$ & {$[0.023,0.024]$} & $\mathrm{A}_{14}$ & {$[0.040,0.041]$} \\
\hline $\mathrm{A}_{14}$ & {$[0.017,0.018]$} & $\mathrm{A}_{23}$ & {$[0.037,0.038]$} \\
\hline $\mathrm{A}_{16}$ & {$[0.012,0.013]$} & $\mathrm{A}_{16}$ & {$[0.029,0.030]$} \\
\hline $\mathrm{A}_{22}$ & {$[0.012,0.013]$} & $\mathrm{A}_{22}$ & {$[0.029,0.029]$} \\
\hline
\end{tabular}


The ranking obtained with the fuzzy TOPSIS shows alternative $\mathrm{A}_{13}$ as the best solution given the preferences of the decision maker:

$\mathrm{A}_{13}>\mathrm{A}_{6}>\mathrm{A}_{9}>\mathrm{A}_{3}>\mathrm{A}_{4}>\mathrm{A}_{8}=\mathrm{A}_{18}>\mathrm{A}_{7}=\mathrm{A}_{17}=\mathrm{A}_{5}=\mathrm{A}_{11}>\mathrm{A}_{21}>\mathrm{A}_{19}>\mathrm{A}_{20}>\mathrm{A}_{1}>\mathrm{A}_{2}>\mathrm{A}_{15}>\mathrm{A}_{10}=\mathrm{A}_{24}>\mathrm{A}_{1}$ ${ }_{2}>\mathrm{A}_{23}>\mathrm{A}_{14}>\mathrm{A}_{16}=\mathrm{A}_{22}$

The ranking of preferences using the fuzzy asymmetric TOPSIS is the following one:

$\mathrm{A}_{6}>\mathrm{A}_{9}>\mathrm{A}_{13}>\mathrm{A}_{3}>\mathrm{A}_{18}>\mathrm{A}_{19}>\mathrm{A}_{1}>\mathrm{A}_{4}>\mathrm{A}_{8}>\mathrm{A}_{7}>\mathrm{A}_{5}>\mathrm{A}_{11}>\mathrm{A}_{17}>\mathrm{A}_{21}>\mathrm{A}_{20}>\mathrm{A}_{2}>\mathrm{A}_{15}=\mathrm{A}_{12}>\mathrm{A}_{10}>$ $\mathrm{A}_{24}=\mathrm{A}_{14}>\mathrm{A}_{23}>\mathrm{A}_{16}>\mathrm{A}_{22}$

In this case the most desirable alternative is $\mathrm{A}_{6}$. Both rankings present problems in ordering the alternatives. For example, in the ranking of the fuzzy TOPSIS, alternatives $\mathrm{A}_{8}$ and $\mathrm{A}_{18}$, or alternatives $A_{15}$ and $A_{12}$ in the ranking of fuzzy and asymmetric TOPSIS are similar. This problem is solved by considering the minor distance to the top alternative. The ranking of the alternatives obtained in this case is:

$\mathrm{A}_{6}>\mathrm{A}_{9}>\mathrm{A}_{13}>\mathrm{A}_{3}>\mathrm{A}_{18}>\mathrm{A}_{19}>\mathrm{A}_{1}>\mathrm{A}_{4}>\mathrm{A}_{8}>\mathrm{A}_{7}>\mathrm{A}_{5}>\mathrm{A}_{11}>\mathrm{A}_{17}>\mathrm{A}_{21}>\mathrm{A}_{20}>\mathrm{A}_{2}>\mathrm{A}_{15}>\mathrm{A}_{12}>\mathrm{A}_{10}>$ $\mathrm{A}_{24}>\mathrm{A}_{14}>\mathrm{A}_{23}>\mathrm{A}_{16}>\mathrm{A}_{22}$

The two rankings, one obtained with the fuzzy TOPSIS and one obtained with the asymmetric fuzzy TOPSIS approach, are similar. However, the consideration of asymmetric distances allows the introduction of the negative effects of surpassing the ideal. Thus, alternative $A_{13}$ fell to the third position, penalized by the excessive frequency of exposure.

To test the significant changes in the results, we now consider other weighting schemes for the two goals, awareness and traffic. Table 7 shows the results for nine different combinations of weights.

Table 7. Rankings from different combinations of weights for the two goals

\begin{tabular}{|c|c|c|c|c|c|c|c|c|}
\hline $\begin{array}{l}\mathrm{A}=10 \% \\
\mathrm{~T}=90 \%\end{array}$ & $\begin{array}{l}\mathrm{A}=20 \% \\
\mathrm{~T}=80 \%\end{array}$ & $\begin{array}{l}\mathrm{A}=30 \% \\
\mathrm{~T}=70 \%\end{array}$ & $\begin{array}{l}\mathrm{A}=40 \% \\
\mathrm{~T}=60 \%\end{array}$ & $\begin{array}{l}\mathrm{A}=50 \% \\
\mathrm{~T}=50 \%\end{array}$ & $\begin{array}{l}\mathrm{A}=60 \% \\
\mathrm{~T}=40 \%\end{array}$ & $\begin{array}{l}\mathrm{A}=70 \% \\
\mathrm{~T}=30 \%\end{array}$ & $\begin{array}{l}\mathrm{A}=80 \% \\
\mathrm{~T}=20 \%\end{array}$ & $\begin{array}{l}\mathrm{A}=90 \% \\
\mathrm{~T}=10 \%\end{array}$ \\
\hline $\mathrm{A}_{6}$ & $\mathrm{~A}_{6}$ & $\mathrm{~A}_{6}$ & $\mathrm{~A}_{6}$ & $\mathrm{~A}_{6}$ & $\mathrm{~A}_{6}$ & $\mathrm{~A}_{6}$ & $\mathrm{~A}_{6}$ & $\mathrm{~A}_{6}$ \\
\hline $\mathrm{A}_{9}$ & $\mathrm{~A}_{9}$ & $\mathrm{~A}_{9}$ & $\mathrm{~A}_{9}$ & $\mathrm{~A}_{9}$ & $\mathrm{~A}_{9}$ & $\mathrm{~A}_{9}$ & $\mathrm{~A}_{9}$ & $\mathrm{~A}_{9}$ \\
\hline $\mathrm{A}_{13}$ & $\mathrm{~A}_{13}$ & $\mathrm{~A}_{13}$ & $\mathrm{~A}_{13}$ & $\mathrm{~A}_{13}$ & $\mathrm{~A}_{13}$ & $\mathrm{~A}_{13}$ & $\mathrm{~A}_{13}$ & $\mathrm{~A}_{3}$ \\
\hline $\mathrm{A}_{3}$ & $\mathrm{~A}_{3}$ & $\mathrm{~A}_{3}$ & $\mathrm{~A}_{3}$ & $\mathrm{~A}_{3}$ & $\mathrm{~A}_{3}$ & $\mathrm{~A}_{3}$ & $\mathrm{~A}_{3}$ & $\mathrm{~A}_{13}$ \\
\hline $\mathrm{A}_{18}$ & $\mathrm{~A}_{18}$ & $\mathrm{~A}_{18}$ & $\mathrm{~A}_{18}$ & $\mathrm{~A}_{18}$ & $\mathrm{~A}_{18}$ & $\mathrm{~A}_{18}$ & $\mathrm{~A}_{18}$ & $\mathrm{~A}_{18}$ \\
\hline $\mathrm{A}_{19}$ & $\mathrm{~A}_{19}$ & $\mathrm{~A}_{19}$ & $\mathrm{~A}_{19}$ & $\mathrm{~A}_{19}$ & $\mathrm{~A}_{19}$ & $\mathrm{~A}_{19}$ & $\mathrm{~A}_{19}$ & $\mathrm{~A}_{19}$ \\
\hline $\mathrm{A}_{1}$ & $\mathrm{~A}_{1}$ & $\mathrm{~A}_{1}$ & $\mathrm{~A}_{1}$ & $\mathrm{~A}_{1}$ & $\mathrm{~A}_{1}$ & $\mathrm{~A}_{1}$ & $\mathrm{~A}_{1}$ & $\mathrm{~A}_{1}$ \\
\hline $\mathrm{A}_{4}$ & $\mathrm{~A}_{4}$ & $\mathrm{~A}_{4}$ & $\mathrm{~A}_{4}$ & $\mathrm{~A}_{4}$ & $\mathrm{~A}_{4}$ & $\mathrm{~A}_{4}$ & $\mathrm{~A}_{4}$ & $\mathrm{~A}_{4}$ \\
\hline
\end{tabular}




\begin{tabular}{lllllllll}
$\mathrm{A}_{8}$ & $\mathrm{~A}_{8}$ & $\mathrm{~A}_{8}$ & $\mathrm{~A}_{8}$ & $\mathrm{~A}_{8}$ & $\mathrm{~A}_{8}$ & $\mathrm{~A}_{8}$ & $\mathrm{~A}_{8}$ & $\mathrm{~A}_{8}$ \\
$\mathrm{~A}_{7}$ & $\mathrm{~A}_{7}$ & $\mathrm{~A}_{7}$ & $\mathrm{~A}_{7}$ & $\mathrm{~A}_{7}$ & $\mathrm{~A}_{7}$ & $\mathrm{~A}_{7}$ & $\mathrm{~A}_{7}$ & $\mathrm{~A}_{7}$ \\
$\mathrm{~A}_{5}$ & $\mathrm{~A}_{5}$ & $\mathrm{~A}_{5}$ & $\mathrm{~A}_{5}$ & $\mathrm{~A}_{5}$ & $\mathrm{~A}_{5}$ & $\mathrm{~A}_{5}$ & $\mathrm{~A}_{5}$ & $\mathrm{~A}_{5}$ \\
$\mathrm{~A}_{11}$ & $\mathrm{~A}_{11}$ & $\mathrm{~A}_{11}$ & $\mathrm{~A}_{11}$ & $\mathrm{~A}_{11}$ & $\mathrm{~A}_{11}$ & $\mathrm{~A}_{11}$ & $\mathrm{~A}_{11}$ & $\mathrm{~A}_{11}$ \\
$\mathrm{~A}_{17}$ & $\mathrm{~A}_{17}$ & $\mathrm{~A}_{17}$ & $\mathrm{~A}_{17}$ & $\mathrm{~A}_{17}$ & $\mathrm{~A}_{17}$ & $\mathrm{~A}_{17}$ & $\mathrm{~A}_{21}$ & $\mathrm{~A}_{21}$ \\
$\mathrm{~A}_{21}$ & $\mathrm{~A}_{21}$ & $\mathrm{~A}_{21}$ & $\mathrm{~A}_{21}$ & $\mathrm{~A}_{21}$ & $\mathrm{~A}_{21}$ & $\mathrm{~A}_{21}$ & $\mathrm{~A}_{17}$ & $\mathrm{~A}_{17}$ \\
$\mathrm{~A}_{20}$ & $\mathrm{~A}_{20}$ & $\mathrm{~A}_{20}$ & $\mathrm{~A}_{20}$ & $\mathrm{~A}_{20}$ & $\mathrm{~A}_{20}$ & $\mathrm{~A}_{20}$ & $\mathrm{~A}_{20}$ & $\mathrm{~A}_{20}$ \\
$\mathrm{~A}_{2}$ & $\mathrm{~A}_{2}$ & $\mathrm{~A}_{2}$ & $\mathrm{~A}_{2}$ & $\mathrm{~A}_{2}$ & $\mathrm{~A}_{2}$ & $\mathrm{~A}_{2}$ & $\mathrm{~A}_{2}$ & $\mathrm{~A}_{2}$ \\
$\mathrm{~A}_{10}$ & $\mathrm{~A}_{10}$ & $\mathrm{~A}_{10}$ & $\mathrm{~A}_{15}$ & $\mathrm{~A}_{15}$ & $\mathrm{~A}_{15}$ & $\mathrm{~A}_{15}$ & $\mathrm{~A}_{15}$ & $\mathrm{~A}_{15}$ \\
$\mathrm{~A}_{15}$ & $\mathrm{~A}_{15}$ & $\mathrm{~A}_{15}$ & $\mathrm{~A}_{12}$ & $\mathrm{~A}_{12}$ & $\mathrm{~A}_{12}$ & $\mathrm{~A}_{12}$ & $\mathrm{~A}_{12}$ & $\mathrm{~A}_{12}$ \\
$\mathrm{~A}_{12}$ & $\mathrm{~A}_{12}$ & $\mathrm{~A}_{12}$ & $\mathrm{~A}_{10}$ & $\mathrm{~A}_{10}$ & $\mathrm{~A}_{10}$ & $\mathrm{~A}_{10}$ & $\mathrm{~A}_{10}$ & $\mathrm{~A}_{10}$ \\
$\mathrm{~A}_{14}$ & $\mathrm{~A}_{14}$ & $\mathrm{~A}_{24}$ & $\mathrm{~A}_{24}$ & $\mathrm{~A}_{24}$ & $\mathrm{~A}_{24}$ & $\mathrm{~A}_{24}$ & $\mathrm{~A}_{24}$ & $\mathrm{~A}_{24}$ \\
$\mathrm{~A}_{24}$ & $\mathrm{~A}_{24}$ & $\mathrm{~A}_{14}$ & $\mathrm{~A}_{14}$ & $\mathrm{~A}_{14}$ & $\mathrm{~A}_{14}$ & $\mathrm{~A}_{14}$ & $\mathrm{~A}_{14}$ & $\mathrm{~A}_{14}$ \\
$\mathrm{~A}_{23}$ & $\mathrm{~A}_{23}$ & $\mathrm{~A}_{23}$ & $\mathrm{~A}_{23}$ & $\mathrm{~A}_{23}$ & $\mathrm{~A}_{23}$ & $\mathrm{~A}_{23}$ & $\mathrm{~A}_{23}$ & $\mathrm{~A}_{23}$ \\
$\mathrm{~A}_{16}$ & $\mathrm{~A}_{16}$ & $\mathrm{~A}_{16}$ & $\mathrm{~A}_{16}$ & $\mathrm{~A}_{22}$ & $\mathrm{~A}_{22}$ & $\mathrm{~A}_{22}$ & $\mathrm{~A}_{22}$ & $\mathrm{~A}_{22}$ \\
$\mathrm{~A}_{22}$ & $\mathrm{~A}_{22}$ & $\mathrm{~A}_{22}$ & $\mathrm{~A}_{22}$ & $\mathrm{~A}_{16}$ & $\mathrm{~A}_{16}$ & $\mathrm{~A}_{16}$ & $\mathrm{~A}_{16}$ & $\mathrm{~A}_{16}$ \\
\hline
\end{tabular}

We can observe that the correlation between the rankings obtained from different combinations of the two goals, awareness and traffic, is higher than 0.999.

\section{Conclusions}

In this paper we have addressed the problem of the optimization of the investment in an online advertising campaign based on pay per click for a stock exchange brokerage service. A new fuzzy asymmetric TOPSIS approach has been proposed. This new model allows us to order the preferences of the decision maker concerning different alternative keyword sets aimed at maximizing the awareness of and the traffic to a company's website. The TOPSISbased approach is computationally simple, and its underlying concept is rational and comprehensible, thus facilitating its implementation in a computer-based system (Huang 2008).

The main novelty of the proposed approach is the use of a characteristic membership function in the distance function to the ideal when the ideal solution is not a maximum or a minimum solution. An advertising frequency below the ideal is considered to be worse than a frequency above the ideal. However, an excessive frequency can cause fatigue in the user. The proposed asymmetric TOPSIS appears to be a suitable tool to deal with these situations, as it penalizes lacks of frequency and softens light excesses. Further research could explore the use of aggregated opinions of experts to fix the positive and negative ideals and the values of the weighting function to penalize excesses and lacks in relation to the ideal. In this sense experts or other aggregation operators can be considered. 
The proposed approach is also able to incorporate the imprecision and uncertainty of the available data. This is the case of the cookies per week and the OTS provided by the Google AdWords tool.

The empirical case shows the effectiveness of the proposed methodology for online advertising planning and provides meaningful rankings and useful information for companies providing stock exchange brokerage services. It is also possible to extend the use of the proposed model to other companies related to investment, finance or insurance and to Internet advertising managers in general. 


\section{References}

Anderson K (2006) Robin Hood marketing: Stealing corporate savvy to sell just causes. Jossey-Bass, San Francisco, CA.

Brooks N (2004) The Atlas rank report I: How search engine rank impacts traffic. http://www.atlassiteoptimization.com/uploadedFiles/Atlas/Atlas_Institute/Published_Content/ Rank Report.pdf

Behzadian, M. et al. (2012) A state-of the-art survey of TOPSIS applications. Expert Systems with Applications 39(17): 13051-13069.

Ceballos B, Jiménez MTL, Pelta DA, Sánchez JM (2013) El método TOPSIS relativo vs. absoluto. Rect@: Revista Electrónica de Comunicaciones y Trabajos de ASEPUMA 14: 181192.

CNMC (2017) El comercio electrónico supera en España los 5.900 millones de euros en el segundo trimestre de 2016, un 20,3\% más que el año anterior. https://www.cnmc.es/sites/default/files/editor_contenidos/Notas\%20de\%20prensa/2016/2017 0104_NP_CE_2T21016.pdf

Dong W, Charikar M, Li K (2008, July) Asymmetric distance estimation with sketches for similarity search in high-dimensional spaces. In Proceedings of the 31st Annual International ACM SIGIR Conference on Research and Development in Information Retrieval (pp. 123130). Singapore.

Feldman J, Muthukrishnan S, Pal M, Stein C (2007) Budget optimization in search-based advertising auctions. In Proceedings of the 8th ACM Conference on Electronic Commerce (pp. 40-49). San Diego, CA, USA.

Feng J, Bhargava HK, Pennock DM (2007) Implementing sponsored search in web search engines: Computational evaluation of alternative mechanisms. INFORMS J Comput 19: 137.

Fruchter GE, Dou W (2005) Optimal budget allocation over time for keyword ads in web portals. J Optim Theory Appl 124(1): 157-174.

García-Cascales MS, Lamata MT (2012) On rank reversal and TOPSIS method. Math Comput Model 56(5-6): 123-132.

Grappone J, Couzin G (2008) Search engine optimization: An hour a day. John Wiley and Sons, Indianapolis.

Hwang CL, Yoon KP (1981) Multiple Attribute Decision Making Methods and Applications. Springer-Verlag, New York.

Infoadex (2017) Estudio Infoadex de la inversion publicitaria en España 2017. http://infoadex.factoriadigitalpremium.es/infoadex3/documentacion/ESTUDIO-COMPLETO2017.pdf 
ITU (2016) Measuring the information society report 2016. http://www.itu.int/en/ITUD/Statistics/Documents/publications/misr2016/MISR2016-w4.pdf

Kim DJ, Ferrin DL, Rao HR (2008) A trust-based consumer decision-making model in electronic commerce: The role of trust, perceived risk, and their antecedents. Decis Support Syst 44(2): 544-564.

Kumar S, Sethi SP (2009) Dynamic pricing and advertising for web content providers. Eur J Oper Res 197(3): 924-944.

Liao Z, Wong WK (2008) The determinants of customer interactions with Internet-enabled ebanking services. J Oper Res Soc 59(9): 1201-1210.

Merigó JM, Gil-Lafuente AM (2012) Decision-making techniques with similarity measures and OWA operators. SORT Stat Oper Res Trans 36(1): 81-102.

Nadaban S, Dzitacb S, Dzitac I (2016) Fuzzy TOPSIS: A general view. Procedia Comput Sci 91: 823-831.

Özlük Ö, Cholette S (2007) Allocating expenditures across keywords in search advertising. J Revenue Pricing Manag 6(4): 347-356.

Regelson M, Fain D (2006, January) Predicting click-through rate using keyword clusters. In Proceedings of the Second Workshop on Sponsored Search Auctions (Vol. 9623).

Saaty T (1987) Rank generation, preservation, and reversal in the analytic hierarchy decision process. Decis Sci 18(2): 157-177.

Saaty TL, Sagir M (2009) An essay on rank preservation and reversal. Math Comput Model 49(5-6): 1230-1243.

Saaty T, Vargas LG (1984) The legitimacy of rank reversal. OMEGA 12(5): 513-516.

Saaty T, Vargas L (1993) Experiments on rank preservation and reversal in relative measurement. Math Comput Model 17(4-5): 13-18.

Selçuk B, Özlük Ö (2013) Optimal keyword bidding in search-based advertising with target exposure levels. Eur J Oper Res 226(1): 163-172.

Shih HS, Shyur HJ, Lee ES (2007) An extension of TOPSIS for group decision making. Math Comput Model 45(7): 801-813.

Zadeh LA (1965) Fuzzy sets. Inf Control 8: 338-353.

Zhao L, Nagurney A (2008) A network equilibrium framework for Internet advertising: Models, qualitative analysis, and algorithms. Eur J Oper Res 187(2): 456-472.

Zhou Y, Chakrabarty D, Lukose R (2008) Budget constrained bidding in keyword auctions and online knapsack problems. In Proceeding of the 17th International Conference on World Wide Web. 
Table 1. Alternative keyword sets

\begin{tabular}{|c|c|}
\hline Alternatives & Keywords set \\
\hline $\mathrm{A}_{1}$ & financial services, investment services, finance, investment \\
\hline $\mathrm{A}_{2}$ & $\begin{array}{l}\text { broker for investment in stock exchange, platform for investment in stock exchange, stock } \\
\text { market values, companies for investment }\end{array}$ \\
\hline $\mathrm{A}_{3}$ & $\begin{array}{l}\text { investment in the stock exchange, how invest in stock exchange, stock exchange shares, how is } \\
\text { it the stock exchange, the stock exchange }\end{array}$ \\
\hline $\mathrm{A}_{4}$ & $\begin{array}{l}\text { stock exchange broker, investment stock exchange, stock exchange market, stock exchange } \\
\text { quotes, stock exchange forex, options, stock exchange operations, foreign exchange, trading, } \\
\text { stock exchange and trading, CFD, stock exchange platform, stock exchange for beginners }\end{array}$ \\
\hline $\mathrm{A}_{5}$ & $\begin{array}{l}\text { investment in the stock exchange, investment stock exchange, stock exchange, Spain stock } \\
\text { exchange, stock exchange market }\end{array}$ \\
\hline $\mathrm{A}_{6}$ & investment in stock exchange, how investment in stock exchange \\
\hline $\mathrm{A}_{7}$ & stock exchange broker, futures, stock exchange agent, \\
\hline $\mathrm{A}_{8}$ & $\begin{array}{l}\text { stocks in the stock exchange, how to trade, win money in the stock exchange, win in the stock } \\
\text { exchange, how win in the stock exchange }\end{array}$ \\
\hline $\mathrm{A}_{9}$ & $\begin{array}{l}\text { learn to invest in stock market, invest in stock market, invest in stock market beginners, values } \\
\text { in stock market investing, investing in stock market online, In which to invest in stock market }\end{array}$ \\
\hline $\mathrm{A}_{10}$ & $\begin{array}{l}\text { how to invest money in stock exchange, invest in the stock exchange, stock exchange ibex } \\
\text { spanish }\end{array}$ \\
\hline $\mathrm{A}_{11}$ & values of the stock exchange, companies of the stock exchange \\
\hline $\mathrm{A}_{12}$ & stock exchange \\
\hline $\mathrm{A}_{13}$ & buy in stock exchange \\
\hline $\mathrm{A}_{14}$ & stock exchange beginners, stock exchange rookie \\
\hline $\mathrm{A}_{15}$ & invest money in the stock exchange, in stock exchange \\
\hline $\mathrm{A}_{16}$ & how to invest in stock exchange for beginners, how to invest in the stock market \\
\hline $\mathrm{A}_{17}$ & broker forex, broker forex trading, forex broker forex trading \\
\hline $\mathrm{A}_{18}$ & broker of forex \\
\hline $\mathrm{A}_{19}$ & $\begin{array}{l}\text { cfd broker, trading broker, brokers cfds, trading online broker, best broker cfd, forex broker, } \\
\text { broker options, broker foreign exchange, cfd online broker }\end{array}$ \\
\hline $\mathrm{A}_{20}$ & $\begin{array}{l}\text { stock exchange money, stock exchange broker, gold stock exchange, online stock exchange, the } \\
\text { stock exchanges, stock exchange profitability }\end{array}$ \\
\hline $\mathrm{A}_{21}$ & stock exchange \\
\hline $\mathrm{A}_{22}$ & cfd trader \\
\hline $\mathrm{A}_{23}$ & real time online stock exchange, real time stock exchange \\
\hline $\mathrm{A}_{24}$ & foreign exchange, stock exchange of \\
\hline
\end{tabular}


Table 2. Decision matrix

\begin{tabular}{|c|c|c|c|c|c|c|c|}
\hline $\begin{array}{l}\text { Keyword } \\
\text { sets }\end{array}$ & Clicks & $\begin{array}{l}\text { Impressions } \\
\text { week }\end{array}$ & $\begin{array}{l}\text { Cookies } \\
\text { week }\end{array}$ & OTS & CTR & $\begin{array}{l}\text { Average } \\
\text { CPC }\end{array}$ & Relevance \\
\hline $\mathrm{A}_{1}$ & 2.920 & 1.123 .077 & {$[100.000,500.000]$} & {$[2.25,11.23]$} & 0.0026 & 0.61 & 10 \\
\hline $\mathrm{A}_{2}$ & 91 & 65.000 & {$[35.000,40.000]$} & {$[1.63,1.86]$} & 0.0014 & 0.50 & 10 \\
\hline $\mathrm{A}_{3}$ & 46.000 & 9.787 .234 & {$[1.500 .000,2.000 .000]$} & {$[4.89,6.52]$} & 0.0047 & 0.10 & 9 \\
\hline $\mathrm{A}_{4}$ & 43.500 & 6.041 .667 & {$[2.000 .000,2.500 .000]$} & {$[2.42,3.02]$} & 0.0072 & 0.10 & 8 \\
\hline $\mathrm{A}_{5}$ & 36.300 & 4.321 .429 & {$[1.500 .000,2.000 .000]$} & {$[2.16,2.88]$} & 0.0084 & 0.07 & 8 \\
\hline $\mathrm{A}_{6}$ & 63.500 & 13.510 .638 & {$[2.500 .000,3.000 .000]$} & {$[4.50,5.40]$} & 0.0047 & 0.11 & 7 \\
\hline $\mathrm{A}_{7}$ & 39.700 & 5.925 .373 & {$[1.500 .000,2.000 .000]$} & {$[2.96,3.95]$} & 0.0067 & 0.06 & 7 \\
\hline $\mathrm{A}_{8}$ & 40.100 & 7.160 .714 & {$[2.000 .000,2.500 .000]$} & {$[2.86,3.58]$} & 0.0056 & 0.09 & 6 \\
\hline $\mathrm{A}_{9}$ & 65.800 & 13.428 .571 & {$[2.000 .000,2.500 .000]$} & {$[5.37,6.71]$} & 0.0049 & 0.10 & 6 \\
\hline $\mathrm{A}_{10}$ & 19 & 1.776 & {$[1.000,1.500]$} & {$[1.18,1.78]$} & 0.0107 & 0.84 & 5 \\
\hline $\mathrm{A}_{11}$ & 42.600 & 4.580 .645 & {$[1.500 .000,2.000 .000]$} & {$[2.29,3.05]$} & 0.0093 & 0.06 & 4 \\
\hline $\mathrm{A}_{12}$ & 361 & 361.000 & {$[200.000,250.000]$} & {$[1.44,1.81]$} & 0.0010 & 0.33 & 4 \\
\hline $\mathrm{A}_{13}$ & 64.100 & 12.094 .340 & {$[250.000,300.000]$} & {$[40.31,48.38]$} & 0.0053 & 0.09 & 4 \\
\hline $\mathrm{A}_{14}$ & 45 & 16.667 & {$[5.000,10.000]$} & {$[1.67,3.33]$} & 0.0027 & 0.50 & 3 \\
\hline $\mathrm{A}_{15}$ & 170 & 106.250 & {$[50.000,100.000]$} & {$[1.06,2.13]$} & 0.0016 & 0.19 & 3 \\
\hline $\mathrm{A}_{16}$ & 9 & 4.737 & {$[2.500,3.000]$} & {$[1.58,1.89]$} & 0.0019 & 0.52 & 2 \\
\hline $\mathrm{A}_{17}$ & 28.100 & 1.243 .363 & {$[250.000,300.000]$} & {$[4.14,4.97]$} & 0.0226 & 0.04 & 1 \\
\hline $\mathrm{A}_{18}$ & 27.300 & 1.087 .649 & {$[200.000,250.000]$} & {$[4.35,5.44]$} & 0.0251 & 0.04 & 1 \\
\hline $\mathrm{A}_{19}$ & 33.200 & 2.862 .069 & {$[500.000,1.000 .000]$} & {$[2.86,5.72]$} & 0.0116 & 0.06 & 1 \\
\hline $\mathrm{A}_{20}$ & 18.000 & 4.090 .909 & {$[1.000 .000,1.500 .000]$} & {$[2.73,4.09]$} & 0.0044 & 0.12 & 1 \\
\hline $\mathrm{A}_{21}$ & 36.900 & 3.324 .324 & {$[1.000 .000,1.500 .000]$} & {$[2.22,3.32]$} & 0.0111 & 0.07 & 1 \\
\hline $\mathrm{A}_{22}$ & 438 & 336.923 & {$[150.000,200.000]$} & {$[1.68,2.25]$} & 0.0013 & 0.52 & 1 \\
\hline $\mathrm{A}_{23}$ & 122 & 33.889 & {$[20.000,25.000]$} & {$[1.36,1.69]$} & 0.0036 & 0.41 & 1 \\
\hline $\mathrm{A}_{24}$ & 42 & 35.000 & {$[15.000,20.000]$} & {$[1.75,2.33]$} & 0.0012 & 0.24 & 1 \\
\hline PIS A+ & 65.800 & 13.510 .638 & {$[2.500 .000,3.000 .000]$} & {$[5,10]$} & 0.025 & 0.04 & 10 \\
\hline NIS A- & 9 & 1776 & {$[1000,1500]$} & {$[0,0]$} & 0.001 & 0.84 & 1 \\
\hline
\end{tabular}


Table 3. Normalized decision matrix

\begin{tabular}{|c|c|c|c|c|c|c|c|c|c|}
\hline \multirow{2}{*}{$\begin{array}{c}\begin{array}{c}\text { Keyword } \\
\text { sets }\end{array} \\
\mathrm{A}_{1}\end{array}$} & \multirow{2}{*}{$\begin{array}{c}\text { Clicks } \\
0.018\end{array}$} & \multirow{2}{*}{$\begin{array}{c}\begin{array}{c}\text { Impressions } \\
\text { week }\end{array} \\
0.040 \\
\end{array}$} & \multicolumn{2}{|c|}{$\begin{array}{c}\text { Cookies } \\
\text { week }\end{array}$} & \multicolumn{2}{|c|}{ OTS } & \multirow{2}{*}{$\begin{array}{l}\text { CTR } \\
0.059\end{array}$} & \multirow{2}{*}{$\begin{array}{c}\begin{array}{c}\text { Average } \\
\text { CPC }\end{array} \\
0.379 \\
\end{array}$} & \multirow{2}{*}{$\begin{array}{c}\text { Relevance } \\
0.383\end{array}$} \\
\hline & & & {$[0.018$} & $0.071]$ & {$[0.053$,} & $0.212]$ & & & \\
\hline $\mathrm{A}_{2}$ & 0.001 & 0.002 & {$[0.006$} & $0.006]$ & {$[0.035$, } & $0.038]$ & 0.032 & 0.311 & 0.383 \\
\hline $\mathrm{A}_{3}$ & 0.279 & 0.344 & {$[0.275$,} & $0.283]$ & {$[0.115$,} & $0.123]$ & 0.106 & 0.062 & 0.345 \\
\hline $\mathrm{A}_{4}$ & 0.264 & 0.213 & {$[0.354$} & $0.367]$ & {$[0.057$,} & $0.057]$ & 0.163 & 0.062 & 0.306 \\
\hline $\mathrm{A}_{5}$ & 0.220 & 0.152 & {$[0.275$} & $0.283]$ & {$[0.051$, } & $0.054]$ & 0.190 & 0.044 & 0.306 \\
\hline $\mathrm{A}_{6}$ & 0.386 & 0.475 & {$[0.425$,} & $0.458]$ & {$[0.102$,} & $0.106]$ & 0.106 & 0.068 & 0.268 \\
\hline $\mathrm{A}_{7}$ & 0.241 & 0.208 & {$[0.275$,} & $0.283]$ & {$[0.070$,} & $0.075]$ & 0.152 & 0.037 & 0.268 \\
\hline $\mathrm{A}_{8}$ & 0.243 & 0.252 & {$[0.354$,} & $0.367]$ & {$[0.067$,} & $0.068]$ & 0.127 & 0.056 & 0.230 \\
\hline $\mathrm{A}_{9}$ & 0.400 & 0.473 & {$[0.354$,} & $0.367]$ & {$[0.126$,} & $0.127]$ & 0.111 & 0.062 & 0.230 \\
\hline $\mathrm{A}_{10}$ & 0.000 & 0.000 & {$[0.000$,} & $0.000]$ & {$[0.028$,} & $0.034]$ & 0.242 & 0.522 & 0.191 \\
\hline $\mathrm{A}_{11}$ & 0.259 & 0.161 & {$[0.275$} & $0.283]$ & {$[0.054$} & $0.058]$ & 0.210 & 0.037 & 0.153 \\
\hline $\mathrm{A}_{12}$ & 0.002 & 0.013 & {$[0.035$} & $0.037]$ & {$[0.034$,} & $0.034]$ & 0.023 & 0.205 & 0.153 \\
\hline $\mathrm{A}_{13}$ & 0.389 & 0.426 & {$[0.042$} & $0.046]$ & {$[0.915$} & $0.947]$ & 0.120 & 0.056 & 0.153 \\
\hline $\mathrm{A}_{14}$ & 0.000 & 0.001 & {$[0.001$,} & $0.001]$ & {$[0.039$} & $0.063]$ & 0.061 & 0.311 & 0.115 \\
\hline $\mathrm{A}_{15}$ & 0.001 & 0.004 & {$[0.009$} & $0.014]$ & {$[0.025$,} & $0.040]$ & 0.036 & 0.118 & 0.115 \\
\hline $\mathrm{A}_{16}$ & 0.000 & 0.000 & {$[0.000$} & $0.000]$ & {$[0.036$} & $0.037]$ & 0.043 & 0.323 & 0.077 \\
\hline $\mathrm{A}_{17}$ & 0.171 & 0.044 & {$[0.042$} & $0.046]$ & {$[0.094$} & $0.097]$ & 0.511 & 0.025 & 0.038 \\
\hline $\mathrm{A}_{18}$ & 0.166 & 0.038 & {$[0.035$} & $0.037]$ & {$[0.102$,} & $0.103]$ & 0.568 & 0.025 & 0.038 \\
\hline $\mathrm{A}_{19}$ & 0.202 & 0.101 & {$[0.092$} & $0.142]$ & {$[0.067$,} & $0.108]$ & 0.262 & 0.037 & 0.038 \\
\hline $\mathrm{A}_{20}$ & 0.109 & 0.144 & {$[0.183$} & $0.212]$ & {$[0.064$} & $0.077]$ & 0.100 & 0.075 & 0.038 \\
\hline $\mathrm{A}_{21}$ & 0.224 & 0.117 & {$[0.183$,} & $0.212]$ & {$[0.052$,} & $0.063]$ & 0.251 & 0.044 & 0.038 \\
\hline $\mathrm{A}_{22}$ & 0.003 & 0.012 & {$[0.028$} & $0.028]$ & {$[0.040$} & $0.042]$ & 0.029 & 0.323 & 0.038 \\
\hline $\mathrm{A}_{23}$ & 0.001 & 0.001 & {$[0.004$,} & $0.004]$ & {$[0.032$} & $0.032]$ & 0.081 & 0.255 & 0.038 \\
\hline $\mathrm{A}_{24}$ & 0.000 & 0.001 & {$[0.003$,} & $0.003]$ & {$[0.041$, } & $0.044]$ & 0.027 & 0.149 & 0.038 \\
\hline PIS A+ & 0.400 & 0.475 & {$[0.425$} & $0.458]$ & {$[0.117$} & $0.189]$ & 0.566 & 0.025 & 0.383 \\
\hline NIS A- & 0.000 & 0.000 & {$[0.000$} & $0.000]$ & {$[0.000$} & $0.000]$ & 0.023 & 0.522 & 0.038 \\
\hline
\end{tabular}


Table 4. Criteria weighting coefficients for each of the goals

\begin{tabular}{cccccccc}
\hline Goal & Clicks & Impressions & Cookies & OTS & CTR & CPC & Relevance \\
\hline Traffic & 0.185 & 0.130 & 0.130 & 0.148 & 0.185 & 0.093 & 0.130 \\
Awareness & 0.123 & 0.175 & 0.175 & 0.140 & 0.123 & 0.123 & 0.140 \\
\hline
\end{tabular}


Table 5. Distances to the PIS and NIS and proximities to the ideal solution

\begin{tabular}{|c|c|c|c|c|c|c|c|}
\hline \multirow[t]{2}{*}{$\begin{array}{c}\text { Keyword } \\
\text { sets }\end{array}$} & \multicolumn{3}{|c|}{ Fuzzy TOPSIS } & \multicolumn{4}{|c|}{ Fuzzy and asymmetric TOPSIS } \\
\hline & $d_{i}\left(A, A^{-}\right)$ & $d_{i}\left(A, A^{+}\right)$ & $R_{i}$ & $d_{i}\left(A, A^{-}\right)$ & $d_{i}\left(A, A^{+}\right)$ & 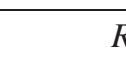 & $R_{i}$ \\
\hline $\mathrm{A}_{1}$ & {$[0.003,0.003]$} & {$[0.029,0.037]$} & {$[0.079,0.081]$} & {$[0.411,0.569]$} & {$[0.397,0.438]$} & {$[0.509$} & $0.565]$ \\
\hline $\mathrm{A}_{2}$ & {$[0.003,0.003]$} & {$[0.038,0.039]$} & {$[0.064,0.067]$} & {$[0.072,0.074]$} & {$[0.972,0.982]$} & {$[0.069$,} & $0.070]$ \\
\hline $\mathrm{A}_{3}$ & {$[0.011,0.011]$} & {$[0.020,0.021]$} & {$[0.346,0.362]$} & {$[0.661,0.663]$} & {$[0.254,0.266]$} & {$[0.713$} & $0.722]$ \\
\hline $\mathrm{A}_{4}$ & {$[0.010,0.010]$} & {$[0.022,0.023]$} & {$[0.310,0.318]$} & {$[0.238,0.241]$} & {$[0.806,0.815]$} & {$[0.228$} & $0.228]$ \\
\hline $\mathrm{A}_{5}$ & {$[0.008,0.008]$} & {$[0.023,0.024]$} & {$[0.256,0.267]$} & {$[0.216,0.217]$} & {$[0.827,0.839]$} & {$[0.206$} & $0.207]$ \\
\hline $\mathrm{A}_{6}$ & {$[0.017,0.018]$} & {$[0.019,0.020]$} & {$[0.468,0.469]$} & {$[0.688,0.705]$} & {$[0.210,0.214]$} & {$[0.766$,} & $0.767]$ \\
\hline $\mathrm{A}_{7}$ & {$[0.008,0.009]$} & {$[0.022,0.024]$} & {$[0.266,0.278]$} & {$[0.220,0.221]$} & {$[0.823,0.835]$} & {$[0.210$} & $0.211]$ \\
\hline $\mathrm{A}_{8}$ & {$[0.009,0.010]$} & {$[0.022,0.023]$} & {$[0.301,0.294]$} & {$[0.227,0.230]$} & {$[0.817,0.825]$} & {$[0.217$} & $0.218]$ \\
\hline $\mathrm{A}_{9}$ & {$[0.016,0.016]$} & {$[0.019,0.020]$} & {$[0.448,0.459]$} & {$[0.700,0.708]$} & {$[0.220,0.228]$} & {$[0.757$,} & $0.761]$ \\
\hline $\mathrm{A}_{10}$ & {$[0.002,0.002]$} & {$[0.036,0.038]$} & {$[0.041,0.043]$} & {$[0.055,0.055]$} & {$[0.990,1001]$} & {$[0.0$} & $0.052]$ \\
\hline $\mathrm{A}_{11}$ & {$[0.008,0.008]$} & {$[0.023,0.024]$} & {$[0.249,0.260]$} & {$[0.207,0.208]$} & {$[0.837,0.848]$} & {$[0.197$,} & $0.198]$ \\
\hline $\mathrm{A}_{12}$ & {$[0.001,0.001]$} & {$[0.037,0.039]$} & {$[0.036,0.038]$} & {$[0.058,0.059]$} & {$[0.987,0.997]$} & {$[0.0$} & $0.056]$ \\
\hline $\mathrm{A}_{13}$ & {$[0.027,0.029]$} & {$[0.009,0.010]$} & {$[0.743,0.744]$} & {$[0.448,0.45$} & {$[0.171,0.175]$} & {$[0.723$,} & $0.724]$ \\
\hline $\mathrm{A}_{14}$ & {$[0.001,0.001]$} & {$[0.037,0.040]$} & {$[0.017,0.018]$} & {$[0.041,0.044]$} & {$\left[\begin{array}{ll}1.001 & 1.014\end{array}\right]$} & {$[0.040$} & $0.041]$ \\
\hline $\mathrm{A}_{15}$ & {$[0.002,0.002]$} & {$[0.037,0.040]$} & {$[0.049,0.052]$} & {$[0.058,0.060]$} & {$[0.985,0.998]$} & {$[0.056$} & $0.057]$ \\
\hline $\mathrm{A}_{16}$ & {$[0.001,0.001]$} & {$[0.039,0.041]$} & {$[0.012,0.013]$} & {$[0.030,0.032]$} & {$\left[\begin{array}{ll}1014 & 1.024\end{array}\right]$} & {$[0.029$} & $0.030]$ \\
\hline $\mathrm{A}_{17}$ & {$[0.010,0.010]$} & {$[0.025,0.027]$} & {$[0.265,0.276]$} & {$[0.177,0.179]$} & {$[0.867,0.876]$} & {$[0.170$} & $0.170]$ \\
\hline $\mathrm{A}_{18}$ & {$[0.011,0.011]$} & {$[0.025,0.027]$} & {$[0.291,0.304]$} & {$[0.574,0.581]$} & {$[0.326,0.336]$} & {$[0.634$} & $0.638]$ \\
\hline $\mathrm{A}_{19}$ & {$[0.006,0.006]$} & {$[0.024,0.028]$} & {$[0.177,0.190]$} & {$[0.510,0.563]$} & {$[0.342,0.365]$} & {$[0.599$} & $0.607]$ \\
\hline $\mathrm{A}_{20}$ & {$[0.004,0.004]$} & {$[0.028,0.030]$} & {$[0.127,0.129]$} & {$[0.132,0.138]$} & {$[0.907,0.923]$} & {$[0.127$,} & $0.130]$ \\
\hline $\mathrm{A}_{21}$ & {$[0.006,0.007]$} & {$[0.025,0.027]$} & {$[0.198,0.206]$} & {$[0.171,0.176]$} & {$[0.869,0.885]$} & {$[0.164$} & $0.166]$ \\
\hline $\mathrm{A}_{22}$ & {$[0.000,0.000]$} & {$[0.039,0.041]$} & {$[0.012,0.013]$} & {$[0.030,0.031]$} & {$[1.014,1.025]$} & {$[0.029$} & $0.029]$ \\
\hline $\mathrm{A}_{23}$ & {$[0.001,0.001]$} & {$[0.038,0.040]$} & {$[0.023,0.024]$} & {$[0.039,0.040]$} & {$[1.006,1.016]$} & {$[0.037$} & $0.038]$ \\
\hline $\mathrm{A}_{24}$ & {$[0.002,0.002]$} & {$[0.039,0.041]$} & {$[0.039,0.041]$} & {$[0.043,0.044]$} & {$[1.001,1.012]$} & {$[0.041$} & $0.042]$ \\
\hline
\end{tabular}


Table 6. Rankings of proximities to the ideal solution

\begin{tabular}{|c|c|c|c|}
\hline Keywords set & $\begin{array}{c}\text { Proximity } R_{i} \\
\text { (Fuzzy TOPSIS) }\end{array}$ & Keywords set & $\begin{array}{c}\text { Proximity } R_{i} \\
\text { (Fuzzy and asymmetric } \\
\text { TOPSIS) } \\
\end{array}$ \\
\hline $\mathrm{A}_{13}$ & {$[0.743,0.744]$} & $\mathrm{A}_{6}$ & {$[0.766,0.767]$} \\
\hline $\mathrm{A}_{6}$ & {$[0.468,0.469]$} & $\mathrm{A}_{9}$ & {$[0.757,0.761]$} \\
\hline $\mathrm{A}_{9}$ & {$[0.448,0.459]$} & $\mathrm{A}_{13}$ & {$[0.723,0.724]$} \\
\hline $\mathrm{A}_{3}$ & {$[0.346,0.362]$} & $\mathrm{A}_{3}$ & {$[0.713,0.722]$} \\
\hline $\mathrm{A}_{4}$ & {$[0.310,0.318]$} & $\mathrm{A}_{18}$ & {$[0.634,0.638]$} \\
\hline $\mathrm{A}_{8}$ & {$[0.294,0.301]$} & $\mathrm{A}_{19}$ & {$[0.599,0.607]$} \\
\hline $\mathrm{A}_{18}$ & {$[0.291,0.304]$} & $\mathrm{A}_{1}$ & {$[0.509,0.565]$} \\
\hline $\mathrm{A}_{7}$ & {$[0.266,0.278]$} & $\mathrm{A}_{4}$ & {$[0.228,0.228]$} \\
\hline $\mathrm{A}_{17}$ & {$[0.265,0.276]$} & $\mathrm{A}_{8}$ & {$[0.217,0.218]$} \\
\hline $\mathrm{A}_{5}$ & {$[0.256,0.267]$} & $\mathrm{A}_{7}$ & {$[0.210,0.211]$} \\
\hline $\mathrm{A}_{11}$ & {$[0.249,0.260]$} & $\mathrm{A}_{5}$ & {$[0.206,0.207]$} \\
\hline $\mathrm{A}_{21}$ & {$[0.198,0.206]$} & $\mathrm{A}_{11}$ & {$[0.197,0.198]$} \\
\hline $\mathrm{A}_{19}$ & {$[0.177,0.190]$} & $\mathrm{A}_{17}$ & {$[0.170,0.170]$} \\
\hline $\mathrm{A}_{20}$ & {$[0.127,0.129]$} & $\mathrm{A}_{21}$ & {$[0.164,0.166]$} \\
\hline $\mathrm{A}_{1}$ & {$[0.079,0.081]$} & $\mathrm{A}_{20}$ & {$[0.127,0.130]$} \\
\hline $\mathrm{A}_{2}$ & {$[0.064,0.067]$} & $\mathrm{A}_{2}$ & {$[0.069,0.070]$} \\
\hline $\mathrm{A}_{15}$ & {$[0.049,0.052]$} & $\mathrm{A}_{15}$ & {$[0.056,0.057]$} \\
\hline $\mathrm{A}_{10}$ & {$[0.041,0.043]$} & $\mathrm{A}_{12}$ & {$[0.055,0.056]$} \\
\hline $\mathrm{A}_{24}$ & {$[0.039,0.041]$} & $\mathrm{A}_{10}$ & {$[0.052,0.052]$} \\
\hline $\mathrm{A}_{12}$ & {$[0.036,0.038]$} & $\mathrm{A}_{24}$ & {$[0.041,0.042]$} \\
\hline $\mathrm{A}_{23}$ & {$[0.023,0.024]$} & $\mathrm{A}_{14}$ & {$[0.040,0.041]$} \\
\hline $\mathrm{A}_{14}$ & {$[0.017,0.018]$} & $\mathrm{A}_{23}$ & {$[0.037,0.038]$} \\
\hline $\mathrm{A}_{16}$ & {$[0.012,0.013]$} & $\mathrm{A}_{16}$ & {$[0.029,0.030]$} \\
\hline $\mathrm{A}_{22}$ & {$[0.012,0.013]$} & $\mathrm{A}_{22}$ & {$[0.029,0.029]$} \\
\hline
\end{tabular}


Table 7. Rankings from different combinations of weights for the two goals

\begin{tabular}{|c|c|c|c|c|c|c|c|c|}
\hline $\begin{array}{l}\mathrm{A}=10 \% \\
\mathrm{~T}=90 \%\end{array}$ & $\begin{array}{l}\mathrm{A}=20 \% \\
\mathrm{~T}=80 \%\end{array}$ & $\begin{array}{l}\mathrm{A}=30 \% \\
\mathrm{~T}=70 \%\end{array}$ & $\begin{array}{l}\mathrm{A}=40 \% \\
\mathrm{~T}=60 \%\end{array}$ & $\begin{array}{l}\mathrm{A}=50 \% \\
\mathrm{~T}=50 \%\end{array}$ & $\begin{aligned} \mathrm{A} & =60 \% \\
\mathrm{~T} & =40 \%\end{aligned}$ & $\begin{array}{l}\mathrm{A}=70 \% \\
\mathrm{~T}=30 \%\end{array}$ & $\begin{array}{l}\mathrm{A}=80 \% \\
\mathrm{~T}=20 \%\end{array}$ & $\begin{array}{l}\mathrm{A}=90 \% \\
\mathrm{~T}=10 \%\end{array}$ \\
\hline $\mathrm{A}_{6}$ & $\mathrm{~A}_{6}$ & $\mathrm{~A}_{6}$ & $\mathrm{~A}_{6}$ & $\mathrm{~A}_{6}$ & $\mathrm{~A}_{6}$ & $\mathrm{~A}_{6}$ & $\mathrm{~A}_{6}$ & $\mathrm{~A}_{6}$ \\
\hline $\mathrm{A}_{9}$ & $\mathrm{~A}_{9}$ & $\mathrm{~A}_{9}$ & $\mathrm{~A}_{9}$ & $\mathrm{~A}_{9}$ & $\mathrm{~A}_{9}$ & $\mathrm{~A}_{9}$ & $\mathrm{~A}_{9}$ & $\mathrm{~A}_{9}$ \\
\hline $\mathrm{A}_{13}$ & $\mathrm{~A}_{13}$ & $\mathrm{~A}_{13}$ & $\mathrm{~A}_{13}$ & $\mathrm{~A}_{13}$ & $\mathrm{~A}_{13}$ & $\mathrm{~A}_{13}$ & $\mathrm{~A}_{13}$ & $\mathrm{~A}_{3}$ \\
\hline $\mathrm{A}_{3}$ & $\mathrm{~A}_{3}$ & $\mathrm{~A}_{3}$ & $\mathrm{~A}_{3}$ & $\mathrm{~A}_{3}$ & $\mathrm{~A}_{3}$ & $\mathrm{~A}_{3}$ & $\mathrm{~A}_{3}$ & $\mathrm{~A}_{13}$ \\
\hline $\mathrm{A}_{18}$ & $\mathrm{~A}_{18}$ & $\mathrm{~A}_{18}$ & $\mathrm{~A}_{18}$ & $\mathrm{~A}_{18}$ & $\mathrm{~A}_{18}$ & $\mathrm{~A}_{18}$ & $\mathrm{~A}_{18}$ & $\mathrm{~A}_{18}$ \\
\hline $\mathrm{A}_{19}$ & $\mathrm{~A}_{19}$ & $\mathrm{~A}_{19}$ & $\mathrm{~A}_{19}$ & $\mathrm{~A}_{19}$ & $\mathrm{~A}_{19}$ & $\mathrm{~A}_{19}$ & $\mathrm{~A}_{19}$ & $\mathrm{~A}_{19}$ \\
\hline $\mathrm{A}_{1}$ & $\mathrm{~A}_{1}$ & $\mathrm{~A}_{1}$ & $\mathrm{~A}_{1}$ & $\mathrm{~A}_{1}$ & $\mathrm{~A}_{1}$ & $\mathrm{~A}_{1}$ & $\mathrm{~A}_{1}$ & $\mathrm{~A}_{1}$ \\
\hline $\mathrm{A}_{4}$ & $\mathrm{~A}_{4}$ & $\mathrm{~A}_{4}$ & $\mathrm{~A}_{4}$ & $\mathrm{~A}_{4}$ & $\mathrm{~A}_{4}$ & $\mathrm{~A}_{4}$ & $\mathrm{~A}_{4}$ & $\mathrm{~A}_{4}$ \\
\hline $\mathrm{A}_{8}$ & $\mathrm{~A}_{8}$ & $\mathrm{~A}_{8}$ & $\mathrm{~A}_{8}$ & $\mathrm{~A}_{8}$ & $\mathrm{~A}_{8}$ & $\mathrm{~A}_{8}$ & $\mathrm{~A}_{8}$ & $\mathrm{~A}_{8}$ \\
\hline $\mathrm{A}_{7}$ & $\mathrm{~A}_{7}$ & $\mathrm{~A}_{7}$ & $\mathrm{~A}_{7}$ & $\mathrm{~A}_{7}$ & $\mathrm{~A}_{7}$ & $\mathrm{~A}_{7}$ & $\mathrm{~A}_{7}$ & $\mathrm{~A}_{7}$ \\
\hline $\mathrm{A}_{5}$ & $\mathrm{~A}_{5}$ & $\mathrm{~A}_{5}$ & $\mathrm{~A}_{5}$ & $\mathrm{~A}_{5}$ & $\mathrm{~A}_{5}$ & $\mathrm{~A}_{5}$ & $\mathrm{~A}_{5}$ & $\mathrm{~A}_{5}$ \\
\hline $\mathrm{A}_{11}$ & $\mathrm{~A}_{11}$ & $\mathrm{~A}_{11}$ & $\mathrm{~A}_{11}$ & $\mathrm{~A}_{11}$ & $\mathrm{~A}_{11}$ & $\mathrm{~A}_{11}$ & $\mathrm{~A}_{11}$ & $\mathrm{~A}_{11}$ \\
\hline $\mathrm{A}_{17}$ & $\mathrm{~A}_{17}$ & $\mathrm{~A}_{17}$ & $\mathrm{~A}_{17}$ & $\mathrm{~A}_{17}$ & $\mathrm{~A}_{17}$ & $\mathrm{~A}_{17}$ & $\mathrm{~A}_{21}$ & $\mathrm{~A}_{21}$ \\
\hline $\mathrm{A}_{21}$ & $\mathrm{~A}_{21}$ & $\mathrm{~A}_{21}$ & $\mathrm{~A}_{21}$ & $\mathrm{~A}_{21}$ & $\mathrm{~A}_{21}$ & $\mathrm{~A}_{21}$ & $\mathrm{~A}_{17}$ & $\mathrm{~A}_{17}$ \\
\hline $\mathrm{A}_{20}$ & $\mathrm{~A}_{20}$ & $\mathrm{~A}_{20}$ & $\mathrm{~A}_{20}$ & $\mathrm{~A}_{20}$ & $\mathrm{~A}_{20}$ & $\mathrm{~A}_{20}$ & $\mathrm{~A}_{20}$ & $\mathrm{~A}_{20}$ \\
\hline $\mathrm{A}_{2}$ & $\mathrm{~A}_{2}$ & $\mathrm{~A}_{2}$ & $\mathrm{~A}_{2}$ & $\mathrm{~A}_{2}$ & $\mathrm{~A}_{2}$ & $\mathrm{~A}_{2}$ & $\mathrm{~A}_{2}$ & $\mathrm{~A}_{2}$ \\
\hline $\mathrm{A}_{10}$ & $\mathrm{~A}_{10}$ & $\mathrm{~A}_{10}$ & $\mathrm{~A}_{15}$ & $\mathrm{~A}_{15}$ & $\mathrm{~A}_{15}$ & $\mathrm{~A}_{15}$ & $\mathrm{~A}_{15}$ & $\mathrm{~A}_{15}$ \\
\hline $\mathrm{A}_{15}$ & $\mathrm{~A}_{15}$ & $\mathrm{~A}_{15}$ & $\mathrm{~A}_{12}$ & $\mathrm{~A}_{12}$ & $\mathrm{~A}_{12}$ & $\mathrm{~A}_{12}$ & $\mathrm{~A}_{12}$ & $\mathrm{~A}_{12}$ \\
\hline $\mathrm{A}_{12}$ & $\mathrm{~A}_{12}$ & $\mathrm{~A}_{12}$ & $\mathrm{~A}_{10}$ & $\mathrm{~A}_{10}$ & $\mathrm{~A}_{10}$ & $\mathrm{~A}_{10}$ & $\mathrm{~A}_{10}$ & $\mathrm{~A}_{10}$ \\
\hline $\mathrm{A}_{14}$ & $\mathrm{~A}_{14}$ & $\mathrm{~A}_{24}$ & $\mathrm{~A}_{24}$ & $\mathrm{~A}_{24}$ & $\mathrm{~A}_{24}$ & $\mathrm{~A}_{24}$ & $\mathrm{~A}_{24}$ & $\mathrm{~A}_{24}$ \\
\hline $\mathrm{A}_{24}$ & $\mathrm{~A}_{24}$ & $\mathrm{~A}_{14}$ & $\mathrm{~A}_{14}$ & $\mathrm{~A}_{14}$ & $\mathrm{~A}_{14}$ & $\mathrm{~A}_{14}$ & $\mathrm{~A}_{14}$ & $\mathrm{~A}_{14}$ \\
\hline $\mathrm{A}_{23}$ & $\mathrm{~A}_{23}$ & $\mathrm{~A}_{23}$ & $\mathrm{~A}_{23}$ & $\mathrm{~A}_{23}$ & $\mathrm{~A}_{23}$ & $\mathrm{~A}_{23}$ & $\mathrm{~A}_{23}$ & $\mathrm{~A}_{23}$ \\
\hline $\mathrm{A}_{16}$ & $\mathrm{~A}_{16}$ & $\mathrm{~A}_{16}$ & $\mathrm{~A}_{16}$ & $\mathrm{~A}_{22}$ & $\mathrm{~A}_{22}$ & $\mathrm{~A}_{22}$ & $\mathrm{~A}_{22}$ & $\mathrm{~A}_{22}$ \\
\hline $\mathrm{A}_{22}$ & $\mathrm{~A}_{22}$ & $\mathrm{~A}_{22}$ & $\mathrm{~A}_{22}$ & $\mathrm{~A}_{16}$ & $\mathrm{~A}_{16}$ & $\mathrm{~A}_{16}$ & $\mathrm{~A}_{16}$ & $\mathrm{~A}_{16}$ \\
\hline
\end{tabular}

